\title{
Design of Tapered Leaky-Wave Antennas in Hybrid Waveguide-Planar Technology for Millimeter Waveband Applications
}

\author{
José Luis Gómez-Tornero, Alejandro de la Torre Martínez, David Cañete Rebenaque, Student Member, IEEE, \\ Marco Gugliemi, and Alejandro Álvarez-Melcón
}

\begin{abstract}
Different types of waveguide leaky-wave antennas, asymmetrically perturbed with printed-circuits, are studied in this paper. The capability to modify the leakage constant of the excited leaky-wave mode, while maintaining unchanged its phase constant, is studied in detail for each design. Several slot and strip configurations are proposed, in which the width and the position of the planar printed circuit perturbation is modified along the length of the antenna to obtain different tapering topologies. All of them are mechanically flexible to realize, thus simplifying the manufacturing process for millimeter wavelengths applications. The two-dimensional method used for the analysis is accurate for any geometry of the planar perturbation, and also allows for the computation of the whole spectrum of modes in these open waveguides. This makes possible to predict the appearance of unwanted channel-guide modes, and also allows for the study of the coupling effect between the desired leaky-wave mode and them. A tapered design is performed and compared with full-wave three-dimension simulations to validate the proposed technology.
\end{abstract}

Index Terms-Hybrid waveguide printed circuit technology, leaky-wave antennas, millimeter wave antennas, sidelobes level control, tapering antennas.

\section{INTRODUCTION}

$\mathbf{L}$ EAKY-WAVE antennas have been studied for the last four decades, exhibiting interesting features due to their traveling-wave nonresonant nature. Among them we can mention frequency scanning capability, very high directivity and large bandwidths. Most of the early leaky-wave antennas (LWA) were based on closed rectangular waveguides, in which some kind of cut or holes were introduced in order to produce the leakage of power along the length of the waveguide [1], [2]. In the last two decades, the research was focused in the millimeter-waveband, where new LWA designs have been proposed to overcome the technological problems associated to these higher frequencies [3]. The main difficulties arise from the higher waveguide losses and from the smaller wavelengths; consequently the new

This work

was supported in part by Spanish National projects ESP2001-4546-PE and TEC2004-04 313-C02-02/TCM and in part by Regional Séneca projects 2002 PB/4/FS/02 and PMPDI-UPCT-2004.

J. L. Gómez-Tornero, D. C. Rebenaque, and A. Álvarez-Melcón are with the Departamento de Tecnologías de la Información y las Comuniaciones, Universidad Politécnica de Cartagena, 30202 Cartagena (Murcia), Spain (e-mail: josel.gomez@upct.es).

A. de la Torre Martínez is with the Mobile Network Engineering Division, Siemens Company, Madrid 50010, Spain.

M. Gugliemi is with RF System Division of the European Space Research and Technology Centre (ESTEC), 2201 AG Noordwijk, The Netherlands. antennas have used low-loss open waveguides designed specifically for use at millimeter-wavelengths, as the groove guide, the nonradiative dielectric (NRD) guide, or the dielectric-image guide. An excellent summary of these original designs and their working mechanism can be found in [4]. These waveguides are open to reduce metal losses, and support a bounded mode which does not cause radiation losses. Some kind of asymmetrical perturbation is needed to make the initially bounded mode to radiate.

All these antennas satisfy most of the desirable aspects for a practical LWA in the millimeter-waveband range, that is: to be based on a simple structure, to be easily fed by standard waveguide sources, to have low metal losses, to radiate in a single polarization (with negligible cross-polarization), to permit a wide range of available beamwidths, and to allow to change the beamwidth without affecting the angle of maximum radiation. The last feature is especially important from three different points of view. First, it is useful for design purposes to be able to control the beamwidth and the angle of radiation independently, in order to conform to any given specifications (different beamwidths for the same scan-angle design, and vice versa) [4]. On the other hand, it is important to notice that, in order to control the sidelobes level, a given tapered illumination along the radiating aperture must be accomplished. This is done by varying the attenuation rate of the leaky-mode (which is related to the beamwidth) along the antenna length in a specified way, while maintaining constant the radiation direction, to force all sections of the antenna to radiate at the same angle [5]. The third reason is related to the practical excitation of the desired leaky-wave mode [6]. This is necessary to avoid direct radiation from the feed discontinuity which can otherwise spoil the antenna performance [6]. In any case, it is very important to find the geometrical parameters of the antenna which can control the leakage rate of the excited leaky-mode in a wide range of values (from zero to large leakage values), without affecting its propagation constant (which determines the radiation angle). Given the fact that these antennas are based on radiation by asymmetry, the position and width of the asymmetrical perturbation introduced in the originally non radiative open waveguide, have shown to offer this desirable control of the radiation [4].

However, for the designs entirely based in waveguide technology, the robust waveguide structure must be modified in order to accomplish the desired electrical properties of the antenna. The waveguide structure has to be adapted to obtain 

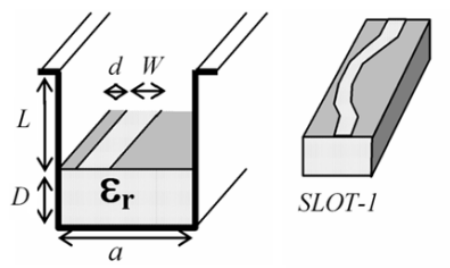

SLOT-1
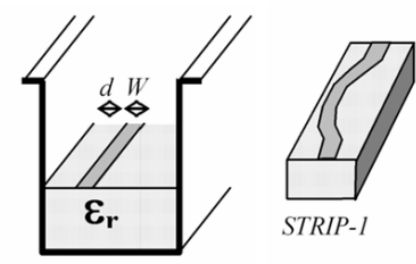

STRIP-I
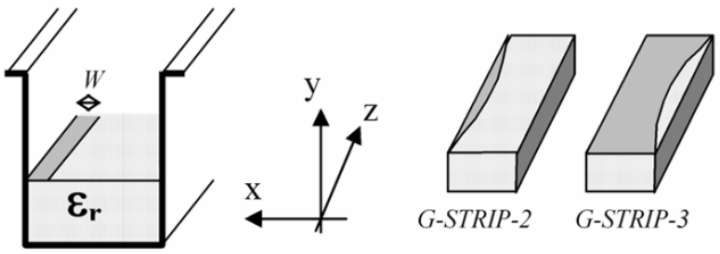

Fig. 1. LWAs for millimeter-waveband applications using hybrid waveguide planar technology, and different tapering techniques, modulating the strip or slot width and position.

a specific tapered profile in order to synthesize the desired beamwidth and radiation direction, therefore resulting in expensive and difficult manufacturing processes. An example can be found in [7] for the case of the taper design of stepped waveguide LWA. A more flexible alternative can be obtained if the waveguide configuration is combined with planar technology, as illustrated for the LWA shown in Fig. 1. The idea is to choose fixed dimensions of the main waveguide $(a, D)$ for a given frequency of operation and scan-angle range, and perturb it by adding some kind of planar metallization. The printed circuit pattern along the length would control the illumination at the aperture, therefore producing the desired beamwidth and sidelobes levels. In this way, by using photolithographic manufacturing processes, it is very easy to change the desired metallization mask, helping to overcome the fabrication difficulties associated to full-waveguide technology, which are even more critical in the millimeter waveband.

This paper describes the electrical features of hybrid waveguide-planar LWA, as those shown in Fig. 1, with special emphasis in the study of the different possible configurations to obtain a versatile tapered design, capable of altering the illumination of the antenna along its length, while maintaining a linear phase variation. As shown in Fig. 1, the different taper topologies studied in this work are based on strip or slot perturbations in the printed side of the dielectric rectangular waveguide, of dimensions $(a, D)$. Different taper options will be presented for each design, by modulating the planar metallization width $W$ or position $d$ around different values, as illustrated in Fig. 1. The different tapering options of these antennas have not been previously studied with so much detail; only in [8], [9] a preliminary study was presented for the slot case, and considering a narrow range of values of the slot width $W$ and position $d$. The lack of a deeper study was due to the approximate nature of the analysis method used in [8.9], based on a small obstacle theory, whose accuracy decreases for large values of the slot width $W$ or position $d$. However, for a practical design of an antenna, it is necessary to accurately obtain the influence of the perturbation (slot or strip) width and position to select the best tapering mechanism for a specific application. For this reason, a fullwave Method of Moments technique specifically conceived for this hybrid waveguide-planar leaky-wave structures [10], [11] is used to obtain the dispersion behavior of the leaky-modes in the proposed LWA. Different full-wave approaches [12]-[14], have been adopted in order to study the same class of printed-circuit antennas. However, the parallel-plate stub, necessary in these LWA to reduce the cross-polarization and focus the radiation in the azimuth angle [4], [11], was assumed to be infinite in [12]-[14]. The effect of the finite stub height ( $L$, according to the schemes in Fig. 1) is critical, since it involves the appearance of channel-guide leaky-modes, which can couple to the desired leaky-wave mode, spoiling the antenna performance [15]. To the authors' knowledge, it is the first time that a full-wave method is applied to study the different possible tapering topologies for LWA in hybrid waveguide printed-circuit technology, taking into account all higher-order effects derived from the finite dimensions of the antenna, and without any restrictions on the geometry, neither for the printed-circuit perturbations (slot and strip width $W$ and position $d$ ) nor for the parallel-plate stub height, $L$.

This paper makes an exhaustive study of the performance of the proposed hybrid technology to conceive leaky-wave antennas for millimeter-wave applications. Section II presents a parametric study of the dispersion behavior of the desired leakymode by changing the slot or strip width and position. As a result, it is determined which taper topology is the best suited to modify the leakage constant of the leaky-mode while maintaining unchanged its phase constant, as previously explained. Section III makes a deep study in the effect of the stub length in the different tapering topologies, showing both the influence of coupling with unwanted leaky-modes (channel-modes [15] and slot-mode [16]) and the control of the polarization purity of the antenna. To obtain these parametric results, a full-wave two-dimensional technique developed in [10], [11] is used. This information is used in Section IV to present a complete tapering procedure at $50 \mathrm{GHz}$, furnishing a longitudinal shaping of the printed circuit to obtain a cosine illumination. The radiation patterns are derived using the information from the two-dimensional dispersion curves, and they are compared to the results obtained from direct analysis of the complete three-dimensional taper structure using a commercial full-wave software (HFSS). Excellent agreement is shown, therefore validating our results. A final discussion is made on the necessity of tapering at the same time both the width and position of the planar metallization to correct the possible phase aberrations in the illumination of the antenna.

\section{WAVEguide PRINTED-Circuit TAPERING TOPOLOGIES}

\section{A. Leaky-Wave Mode Analysis}

The study of the radiation properties of LWA can be easily treated by determining the complex longitudinal propagation constant of the excited leaky-wave mode in the antenna. This 


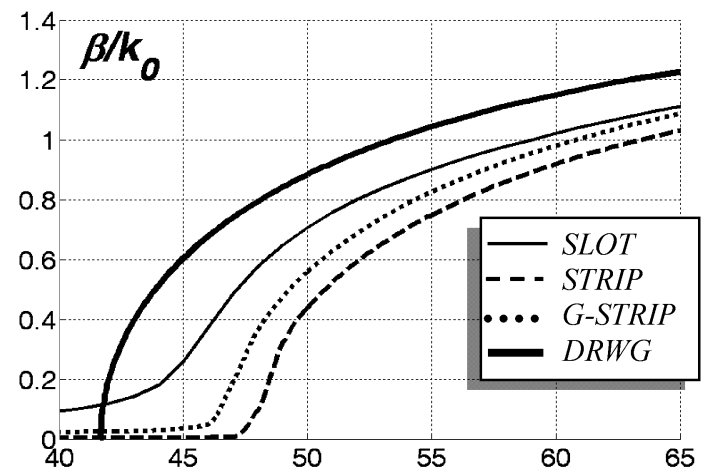

a)

freq $(\mathrm{GHz})$

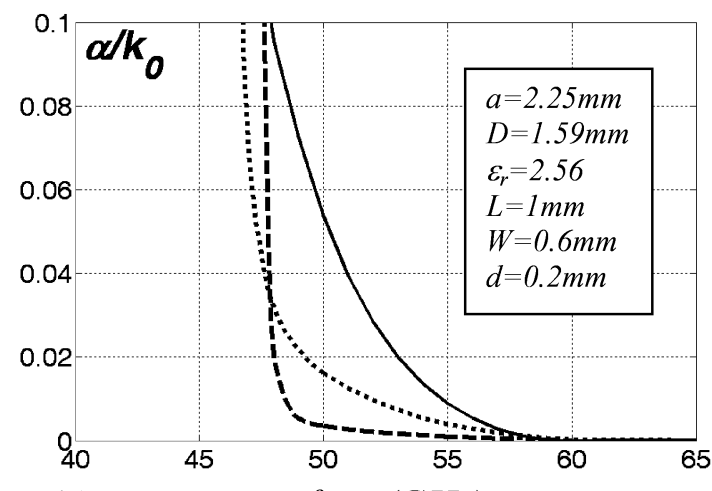

b)

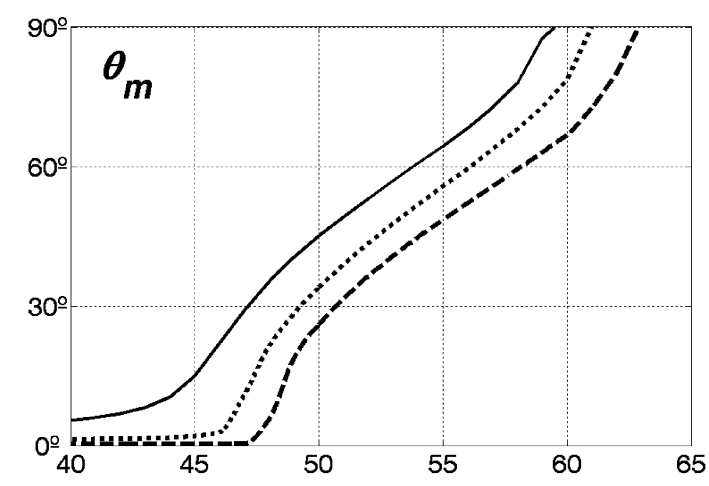

c) freq $(\mathrm{GHz})$

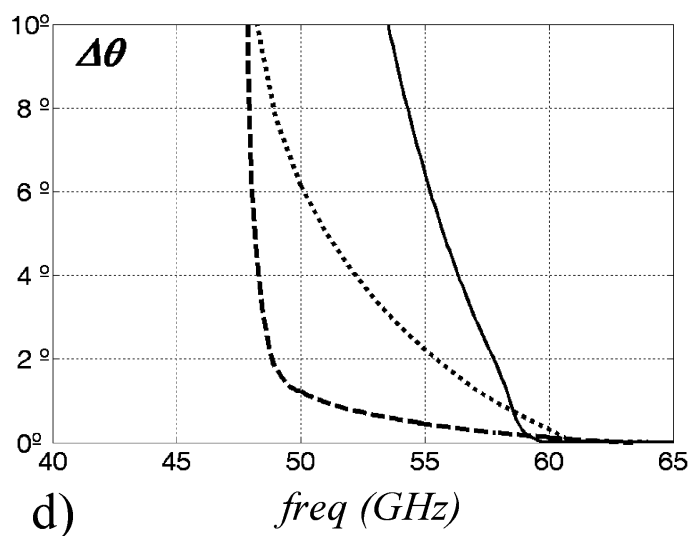

d)

Fig. 2. Frequency dispersion curves of the three types of leaky-wave antennas.

propagation constant is formed by the phase $(\beta)$ and the attenuation $(\alpha)$ parts [4]

$$
k=\beta-j \alpha
$$

where the attenuation part is related to the radiation losses rate $(\mathrm{Np} / \mathrm{m})$. From the phase and attenuation constants, the angle of maximum radiation, $\theta_{m}$ (measured from the broadside direction), and the $3 \mathrm{~dB}$ beamwidth $(\Delta \theta)$ can be approximately determined with the next analytical expressions [4], where $L_{A}$ corresponds to the antenna length

$$
\begin{gathered}
\sin \theta_{m} \cong \frac{\beta}{k_{0}} \\
\Delta \theta \cong \frac{1}{\frac{L_{A}}{\lambda_{0}} \cos \theta_{m}} \approx \frac{\alpha / k_{0}}{0.183 \cdot \cos \theta_{m}} .
\end{gathered}
$$

In (3), the length of the antenna $\left(L_{A}\right)$ has been chosen so that $90 \%$ of the power is radiated, while the remaining $10 \%$ is absorbed by a matched load. From these equations, it is understood that, once $\beta$ and $\alpha$ are known as a function of the antenna geometry or the frequency, all the antenna radiation properties can be easily determined for a given antenna dimensions, or alternatively, the antenna geometry can be designed to conform to the desired specifications. For a more versatile design, it would be desirable to choose the pointing direction of the antenna $\left(\theta_{m}\right)$ and its beamwidth $(\Delta \theta)$ independently, which means to vary $\alpha$ in a large range of values without affecting to the value of $\beta$. Moreover, the aperture amplitude distribution of a LWA must be tapered in some specified fashion to reduce and control the sidelobes of the radiation pattern. To produce the taper needed for the sidelobes requirements, it is necessary to vary the value of $\alpha$ of the leaky-wave mode along the length $\left(L_{A}\right)$ of the antenna. The variation of $\alpha$ as a function of the antenna longitudinal position- $\alpha(z)$ - to obtain a given aperture illumination, can be found in [4]. In any case, $\beta$ must be kept constant along the length of the tapered antenna, so that all parts of the antenna aperture radiate at the same angle $\theta_{m}$ (otherwise phase aberrations are produced). Therefore, the variation of the normalized phase and leakage constants of the desired leaky-mode must be examined as a function of the different geometrical parameters of the antenna cross section, in order to find the way to alter the value of $\alpha$ while maintaining $\beta$ unchanged.

To perform this study, a full-wave space-domain Green's function method of moments (MoM) technique, specifically developed for the analysis of leaky-wave modes in multilayered hybrid planar-waveguide structures, is used for the analysis of the antennas shown in Fig. 1. The method is described with detail in [10] and [11], where it is shown good accuracy by comparisons with other full-wave techniques (spectral domain), while maintaining a strong analytical nature, thus providing fast computation of the dispersion curves. It is worth mentioning here that this method develops rigorous electric and magnetic field integral equations (EFIE and MFIE) to model the currents in the strips and slots planar discontinuities, taking into account all higher order effects. In this way, the two-dimensional eigenvalue problem is solved to obtain the phase and attenuation constants of the leaky-modes of the structure. This method does not make any assumptions on the geometry or the mode which is propagating, therefore being able to find the whole spectrum 


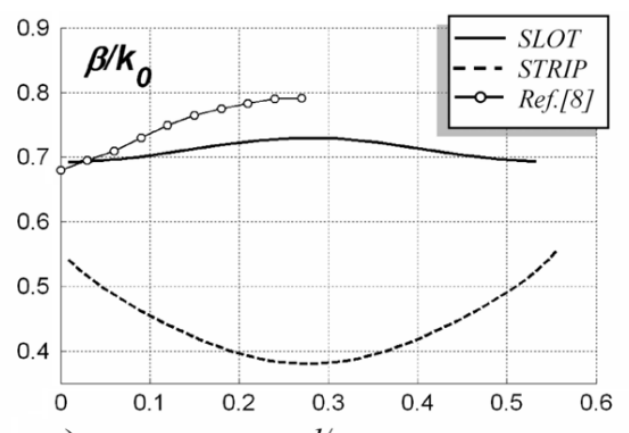

a)
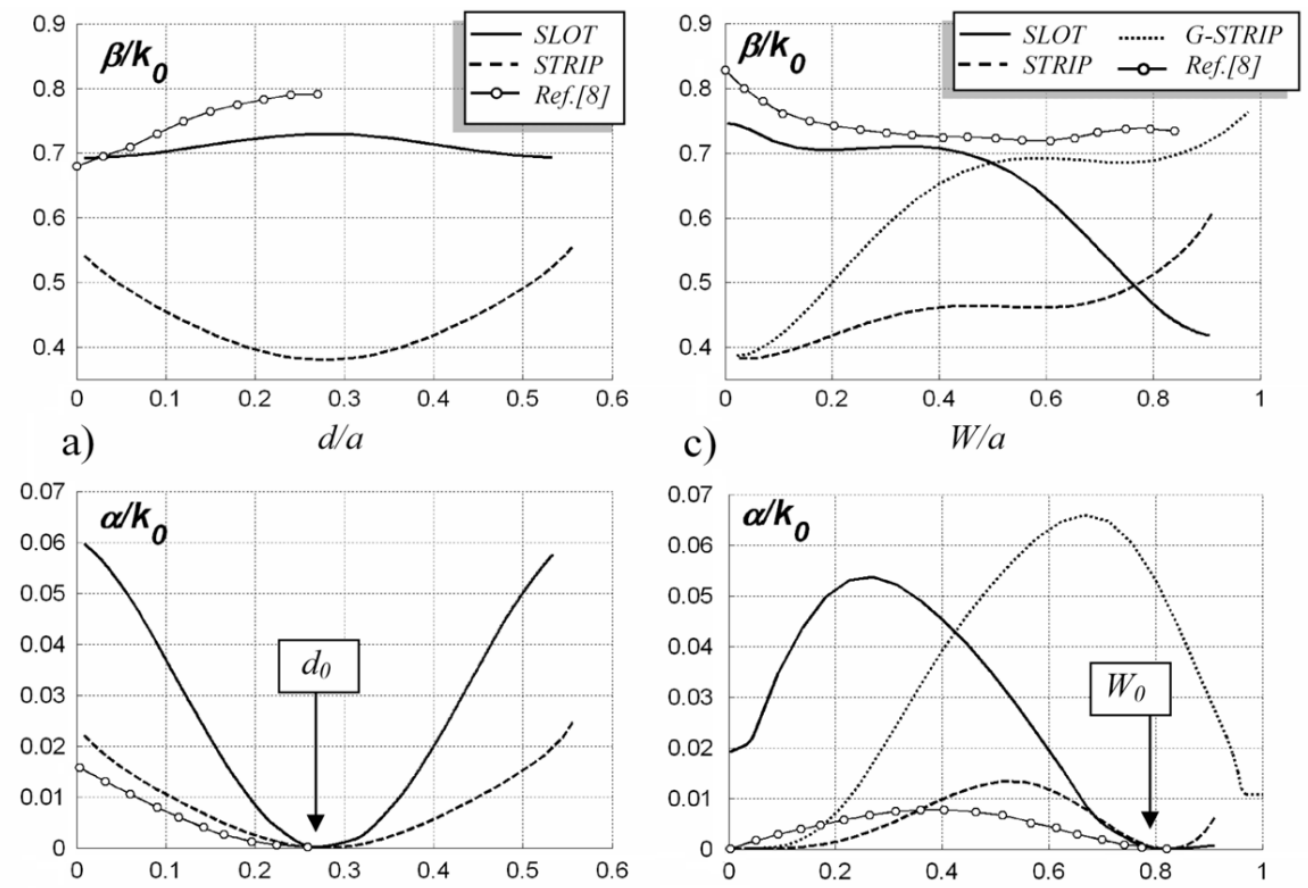

b)

$d / a$

0.07

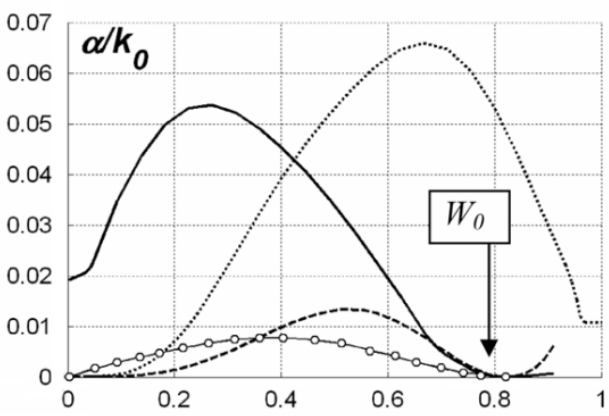

d)

$W / a$

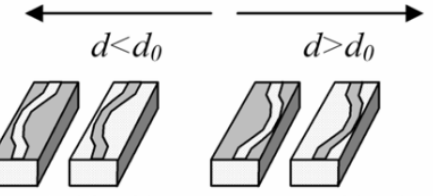

$\longrightarrow \stackrel{W / a}{\longleftarrow}$

$W>0$

$W<W$
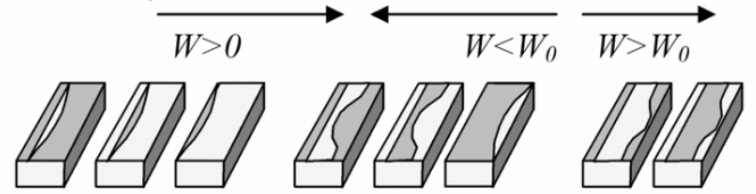

TAPER-1 $\left(d<d_{0}\right)$ TAPER-1 $\left(d>d_{0}\right)$

TAPER-2 $(W>0) \quad$ TAPER-3 $\left(W<W_{0}\right)$ TAPER- $4\left(W>W_{0}\right)$

Fig. 3. Control of $\alpha$ with the perturbation relative offset and width for the three types of printed-circuit LWA $\left(a=2.25 \mathrm{~mm}, D=1.59 \mathrm{~mm}, \varepsilon_{r}=2.56, L=1\right.$ $\mathrm{mm}, \boldsymbol{W}=\mathbf{1} \mathrm{mm}, \boldsymbol{d}=\mathbf{0 . 2} \mathrm{mm}, f=50 \mathrm{GHz}$ ) and comparisons with [8]. Also the different taper topologies corresponding to each range of variation are presented at the bottom of the figure.

of modes in the antenna for any frequency and geometrical parameters. This is of special interest, since the appearance of unwanted modes can spoil the performance of the antenna if they are excited, or if they couple with the desired leaky-wave mode, as mentioned in previous works [15], [16].

Three types of printed circuit perturbations are analyzed, namely the slot-type, the strip-type and the grounded-strip type, which can be seen in Fig. 1. All these LWA are based on the dielectric filled rectangular waveguide main mode $\left(\mathrm{TE}_{10}\right.$ mode in the rectangular waveguide of width $a$ and height $D$, shown in Fig. 1), which is perturbed by adding a slot in its wide wall (or alternatively, by removing the wide wall and adding a strip in the dielectric interface). For this reason, the dimensions of the rectangular waveguide must be fixed for a given frequency of operation. The radiation region of the perturbed leaky-wave mode will be located above the cut-off frequency of this $\mathrm{TE}_{10}$ mode, whose well-known and analytical dispersion curve can be used as an initial approximation to control the operating frequency of these antennas. Fig. 2(a) shows the normalized phase constant of the $\mathrm{TE}_{10}$ leaky-wave mode obtained from the perturbation of the rectangular waveguide main mode for each antenna. Also the $\mathrm{TE}_{10}$ main mode of the dielectric-filled rectangular waveguide (DRWG) has been plotted for reference. However, Fig. 2(a) shows the necessity to compute the rigorous phase constant of the leaky-mode for an analysis of the correct beam direction of each antenna. As expected, the strip (and grounded strip) LWA differs more from the DRWG solution than the slot case, since the perturbation introduced to the top wall is stronger for the strips. Nevertheless, these three types of antennas seem to be valid to operate in the same frequency rage, from 50 to $60 \mathrm{GHz}$, which has been obtained using a rectangular dielectric guide of dimensions $a=2.25 \mathrm{~mm}, D=1.59$ $\mathrm{mm}$, with a relative permittivity $\varepsilon_{r}=2.56$ (following the data used in [8]). On the other hand, in Fig. 2(b) it is shown that the leakage constant is quite different for each antenna ( $\alpha$ is higher for the slot-type than for the strip antennas). As it will be studied, the maximum value of attainable leakage-rate will depend on the particular perturbation dimensions. The geometrical values of these LWA (according to the schemes of Fig. 1) can be seen in the inset of Fig. 2(b). Fig. 2(a) and (b) are replotted in Fig. 2(c) and (d), but replacing $\beta$ and $\alpha$ by $\theta_{m}$ and $\Delta \theta[(2)$ and (3)] to show the frequency scanning (from broadside to forward endfire), and the variation of beamwidth with frequency of these antennas. As it is well-known, dielectric-filled LWA suffer from this variation of $\alpha$ with frequency, which can be a problem for some antenna applications. However, endfire radiation cannot be obtained without a dielectric filled configuration, which also improves the scanning sensitivity behavior with respect to air-filled LWA [4]. The sudden rise of $\alpha$ observed for the lower frequencies in Fig. 2(b) is not due to an increase of the radiation rate, but to the reactive attenuation of the fields below the cut-off frequency [4], [11]. 
Obviously, this cut-off region cannot be used for antenna applications, as the signal does not propagate (nor radiate), but is reflected back to the source. Besides, the upper frequency of the radiation region is determined by the transition frequency of the leaky-mode to a surface-wave mode [17], which makes possible the radiation close to the endfire direction $\left(\theta_{m}=90^{\circ}\right)$. The radiating bandwidth of these inhomogeneous LWA can be limited by the next expression [11]

$$
\frac{\alpha}{k_{0}}<\frac{\beta}{k_{0}}<1
$$

\section{B. Taper Printed-Circuit Topologies}

Once the operating bandwidth of these antennas (and therefore the variation of the radiated beam direction with frequency) has been determined by choosing the proper dielectric rectangular guide (dimensions $a$ and $D$, and substrate permittivity $\varepsilon_{r}$, see Fig. 1), the printed circuit configuration must be analyzed. The planar perturbations (slots or strips) must be capable to control the leakage rate without affecting the pointing of the antenna (phase constant), thus making possible to choose the desired beamwidth and sidelobes level for the same pointing angle $\theta_{m}$. For this purpose, Fig. 3 shows the results obtained for the normalized phase and attenuation constant of the desired leaky-wave mode (at the frequency of $50 \mathrm{GHz}$ ), by varying the width and position of the photoetched perturbation for each antenna design (slot, strip, and grounded strip). The results obtained in [8] for the slot-type LWA are also plotted with round marks in Fig. 3 for comparison purposes. In [8], the dispersion curves for the slot width modulation were plotted using $\theta_{m}$ and $\Delta \theta$ instead of $\beta / k_{0}$ and $\alpha / k_{0}$; however, (2)-(3) provide the straightforward relation. It is important to notice that the small obstacle perturbation used in [8] is not precise for $W>0.25 a$ or $d>0.1 a$, as commented in [11]. This is the reason why $\beta$ becomes different from our results above these values. Also it must be mentioned that the difference in the values of $\alpha$ (our results show higher values of $\alpha$ than [8]) are due to two different reasons. First, as it was demonstrated in [11], full-wave techniques (in the Spectral Domain and in the Space Domain) give a higher value of $\alpha$ than the single mode TRT used in [8]. Second, a practical LWA must have a finite parallel-plate stub length, $L$. In our case, $L$ is equal to $1 \mathrm{~mm}$; but in [8] simulations were made for an infinite stub. The influence of the finite stub analysis has been reported in many works [11], [15], showing a quasiperiodic variation of $\alpha$, with peaks and valleys around the infinite length results. Nevertheless, the same dependence of $\alpha$ with $W$ and $d$ is observed in Fig. 3 for both methods, with the nulls of $\alpha$ occurring at the same slot position $d_{0}$ and width $W_{0}$.

The relative slot or strip offset, $d / a$, is varied in Fig. 3(a) and (b), for a constant perturbation width $(W=1 \mathrm{~mm})$. It can be seen how the leakage rate $\alpha$ can be smoothly varied from large values for a highly asymmetrical location of the perturbation $(d=0)$, to zero radiation when the slot or strip is symmetrically located $\left(d=d_{0}\right)$. The value of this symmetry-case position can be computed as

$$
\frac{d_{0}=a-W}{2 .}
$$

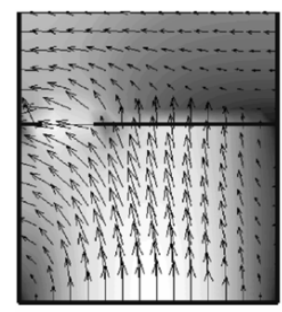

$W / a=0.2$

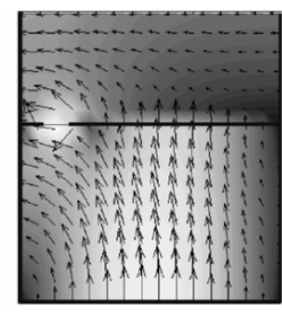

$W / a=0.1$

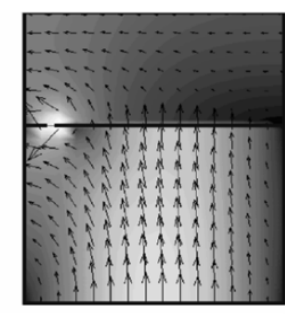

$W / a=0.03$
Fig. 4. Leaky-wave mode transverse electric fields for different slot widths at $50 \mathrm{GHz}(d=0.2 \mathrm{~mm})$.

For the dimensions of the antennas ( $a=2.25 \mathrm{~mm}, W=1$ $\mathrm{mm})$, the value of the nonradiation position is equal to $d_{0}=$ $0.625 \mathrm{~mm}\left(d_{0} / a=0.2778\right)$, as can be checked in Fig. 3(b) for the abscissa point where $\alpha$ becomes zero. Fig. 3(a) shows that $\beta$ suffers a small variation around this value of $d=d_{0}$. This variation seems to be higher for the strip case than for the slot case. Another difference between these two antennas is that the slot-type seems to permit a higher value of maximum radiation rate $\left(\alpha / k_{0}=0.06\right.$ for $\left.d=0\right)$ than the strip-type $\left(\alpha / k_{0}=\right.$ 0.022 for $d=0$ ). However, the behavior of the dispersion curves with $d$ might depend on the fixed value of $W$ (in this particular case, we have chosen $W=1 \mathrm{~mm}$ ). This type of control of the radiation leads to the first taper configuration, which consist in a modulation of the strip or slot position around $d_{0}$, as illustrated at bottom of Fig. 3(b) (TAPER-1, $d<d_{0}$ ). It must be noted that the response of the taper is the same for values of $d$ below or above $d_{0}$. This tapering process was also shown in Fig. 1 (SLOT-1 and STRIP-1), and cannot be applied to the groundedstrip antenna since for the g-strip geometry, the position of the strip is fixed $(d=0)$.

Another type of taper mechanism can be achieved by modulating the strip or slot width $(W)$ for a constant position $(d=0.2$ $\mathrm{mm})$. In this case, there are two ways to make $\alpha$ equal to zero, and then to increase it. The first is a variation of the perturbation around the zero width $(W>0)$, which means that the perturbation does not exist, and therefore the main bounded mode of the open waveguide keeps being nonradiative. This situation leads to the TAPER-2 type for the three studied LWA, as shown below Fig. 3(d). It can be seen from Fig. 3(c) and (d), that for the slot-type and strip-type LWA, $\beta$ is barely affected around $W=0$, while $\alpha$ is varied to a maximum value. This maximum value is higher for the slot-case and occurs for a smaller width than for the strip case. It is interesting to observe the behavior of the slot case when the slot width tends to zero. In principle, one would say that as the slot width tends to zero, the phase constant of the leaky-mode should tend to that of the $\mathrm{TE}_{10}$ mode of the completely closed dielectric rectangular guide. At the same time, $\alpha$ should tend to zero, as it would correspond to a completely closed waveguide. But, as it can be seen in Fig. 3(d), the leakage rate tends to a nonzero value when the slot widths is made very small $\left(\alpha / k_{0} \approx 0.02\right)$.

This phenomenon can be explained from the leaky-mode fields' behavior. Fig. 4 shows the evolution of the transverse electric field for a slot offset $d=0.2 \mathrm{~mm}$, and different widths $(W / a=0.2, W / a=0.1$, and $W / a=0.03$ ). It can be seen from Fig. 4 that the narrow slot creates a high dispersion of the field lines outside the dielectric guide, providing lower values 

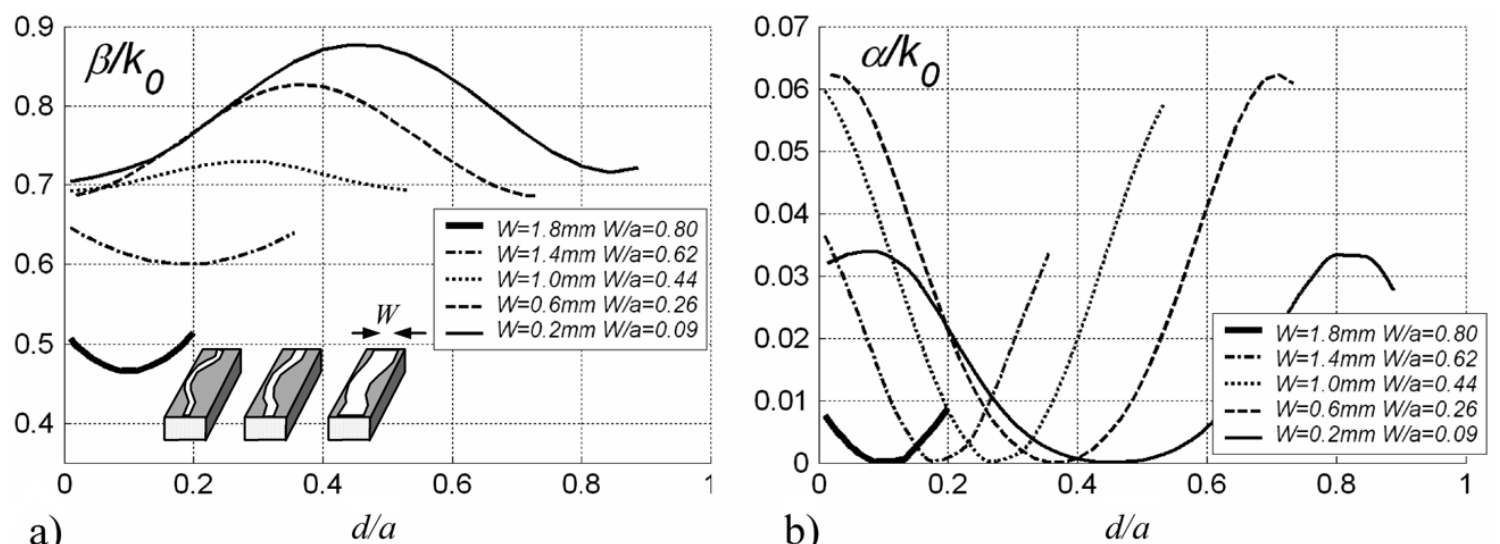

Fig. 5. Position-modulated taper for the slot-type LWA (SLOT-1) for different slot widths $\left(a=2.25 \mathrm{~mm}, D=1.59 \mathrm{~mm}, \varepsilon_{r}=2.56, L=1 \mathrm{~mm}, f=50 \mathrm{GHz}\right)$.

of $\beta$ than the completely closed guide, as well as a nonzero value of the leakage rate. This is due to the fact that there is no electrical contact between the two sides of the slot. Since there is no electrical contact, there is no current flowing from one side of the slot to the other. A zero leakage rate would correspond to the closed structure, where there is an electrical contact between the two sides of the slot, allowing the currents to flow. The same phenomenon occurs for the grounded strip, when the strip width is made very wide, so that there is a small gap in one side of the guide (TAPER-3 in Fig. 3). In this case there is no electrical contact between this side of the strip and the side wall of the guide. For this reason, the slot width modulation around $W=0$ (SLOT-2 in Fig. 1) and the wide grounded strip (G-STRIP-3 in Fig. 1) cannot be used as a taper topology for the antennas proposed in this paper. However, the strip allows for a null radiation rate when the strip width is made zero, as it can be seen in Fig. 3(d) $\left(\alpha / k_{0}\right.$ tends to zero for very narrow strips). The narrow strip does not create much perturbation in the bounded mode of the nonradiative dielectric guide, achieving null radiation when the strip width tends to zero.

The second mechanism that can lead to zero radiation is due to a value of the width which makes the perturbation symmetrically located. This condition can be computed using (5), leading to: $W_{0}=a-2 d$. For the slot and strip antennas studied ( $a=$ $2.25 \mathrm{~mm}, d=0.2 \mathrm{~mm}$ ), this value corresponds to the width $W=1.85 \mathrm{~mm}(W / a=0.822)$, which is in Fig. 3(d). Around this value, there are two different ways to taper the structure; the first is by narrowing the perturbation $\left(W<W_{0}\right.$, which is the fundament of TAPER-3 type in Fig. 3), and the second is by making the slot or strip wider ( $W>W_{0}, T A P E R-4$ in Fig. 3). TAPER 3 can be successfully applied to the strip-type antennas, but not for the slot-case, since the slot phase constant shows a strong variation around $W_{0}$ (both for $W>W_{0}$-TAPER4 -and for $\left.W<W_{0}-T A P E R-3-\right)$. Finally, the TAPER-4 procedure $\left(W>W_{0}\right)$ is not convenient neither for the strip nor for the slot, as $\beta$ is highly dispersive with $W$ in this area for both perturbations.

\section{Optimum Printed-Circuit Width and Position.}

In addition, it is necessary to compute with precision the variation which $\beta$ might suffer for any width and position, in order to choose the best taper mechanism. In addition, to aid the design of the final antenna, it is useful to obtain the appropriate range of widths $(W)$ and the best positions of the planar perturbations $(d)$. This can be done by studying the family of curves obtained for $\beta$ and $\alpha$, when $d$ is swept (for different values of $W$ ), and also when $W$ is swept for different positions $d$. In this way, it can be easily obtained the most suitable taper mechanism for each printed circuit configuration (strip, grounded strip, and slot). Fig. 5 shows the results for the normalized $\beta$ and $\alpha$ obtained by modulating the position of the slot (SLOT-1 taper type in Fig. 1) for different slot widths. For each curve (for each width $W), d$ is swept from $d=0$ to $d_{\mathrm{MAX}}$, being $d_{\mathrm{MAX}}$ the maximum variation of the position of the edge of the slot given by

$$
d_{\mathrm{MAX}}=a-W
$$

As $W$ is increased, the sweep range of $d$ is reduced, and also the offset $d=d_{0}$ required for no radiation [given by (6)]. This can be clearly seen in the nulls of $\alpha$ in Fig. 5(b). There are two important aspects relating the slot width that must be chosen to obtain a good tapering. First, there is a value of optimum width ( $\left.W_{\mathrm{OPT}}\right)$ which reduces the variation of $\beta$ in the whole range of modulated positions, $d$. From Fig. 5(a), this value of $W_{\mathrm{OPT}}$ is between $W / a=0.44$ and $W / a=0.62$ (it is expected to be closer to $W / a=0.44$, from the tendency of the curves). The second point relates the ability to vary $\alpha$ to the maximum reachable value (in order to be able to choose between a wide range of beamwidths). Fig. 5(b) shows that there is also an optimum value of width to obtain a maximum range of variation of $\alpha$, which is between $W / a=0.26$ and $W / a=0.44$. It can therefore be said, that the slot width must be chosen around this optimum value of $W / a=0.44$ to obtain the best tapering process by modulating the position of the slot $(W / a=0.44$ provides a maximum $\alpha / k_{0}=0.06$ with an absolute variation in $\beta$ of only $\left.\Delta \beta / k_{0}=0.03\right)$.

Fig. 6 shows the same taper topology, but now applied to the strip LWA (STRIP-1 in Fig. 1). Now, the minimum change of $\beta$ [Fig. 6(a)] is obtained for the narrower strip curve $(W / a=$ 0.09 ), which unfortunately gives the minimum range of variation of $\alpha$ [Fig. 6(b)]. To obtain the maximum value of $\alpha$, one must choose a wider strip $(W / a=0.62)$, which makes $\beta$ to 


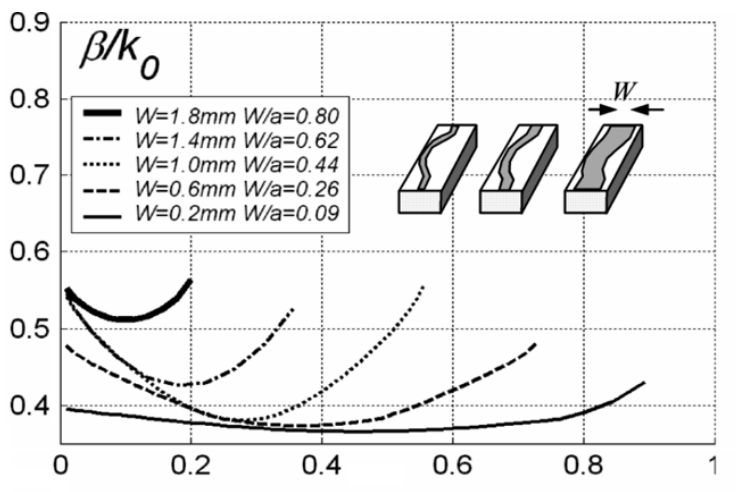

a)

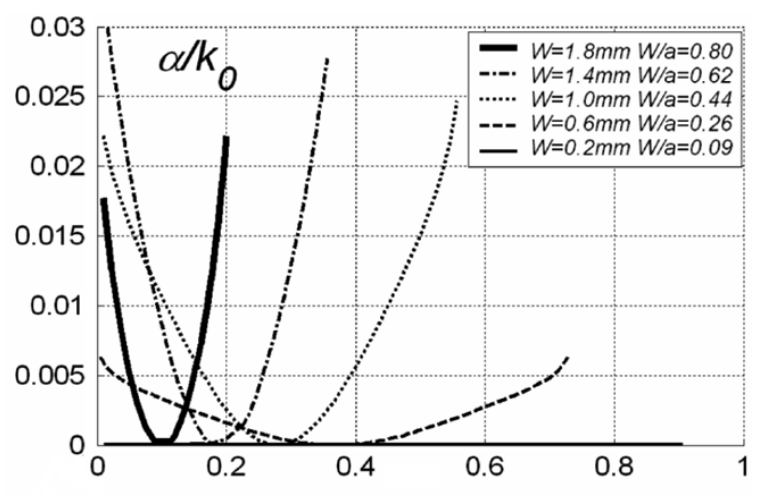

b)

$d / a$

Fig. 6. Position-modulated taper for the strip-type LWA (STIP-1) for different strip widths $\left(a=2.25 \mathrm{~mm}, D=1.59 \mathrm{~mm}, \varepsilon_{r}=2.56, L=1 \mathrm{~mm}, f=50\right.$ $\mathrm{GHz})$.
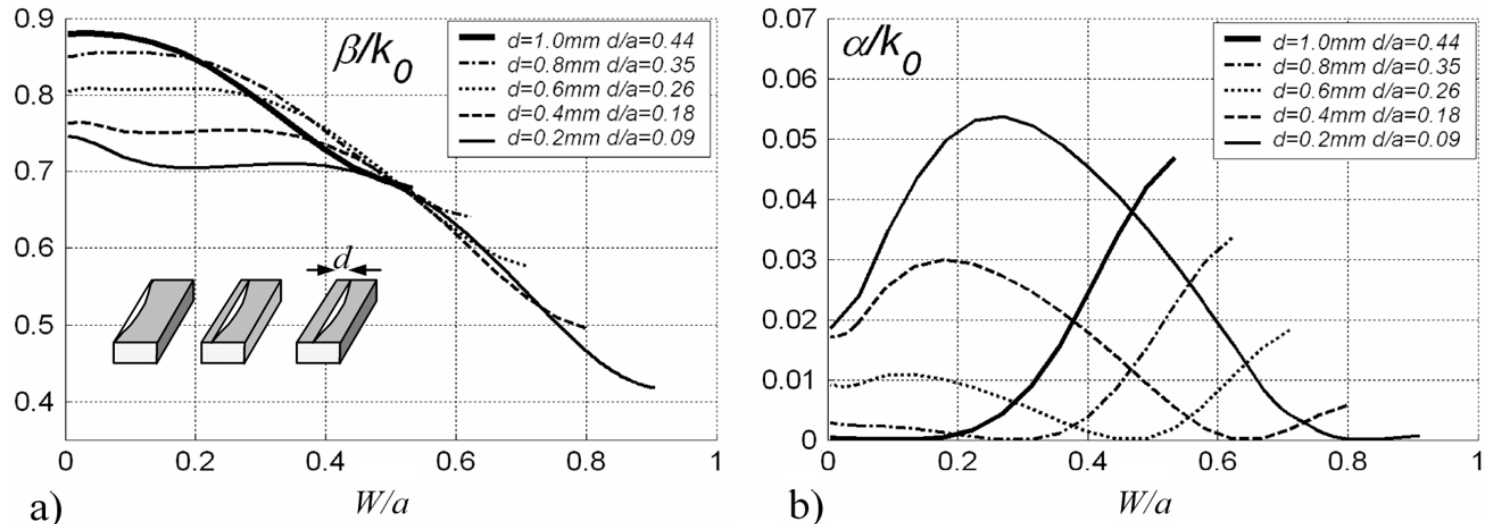

Fig. 7. Width-modulated taper for the slot-type LWA (SLOT-2) for different slot offsets $\left(a=2.25 \mathrm{~mm}, D=1.59 \mathrm{~mm}, \varepsilon_{r}=2.56, L=1 \mathrm{~mm}, f=50 \mathrm{GHz}\right)$.

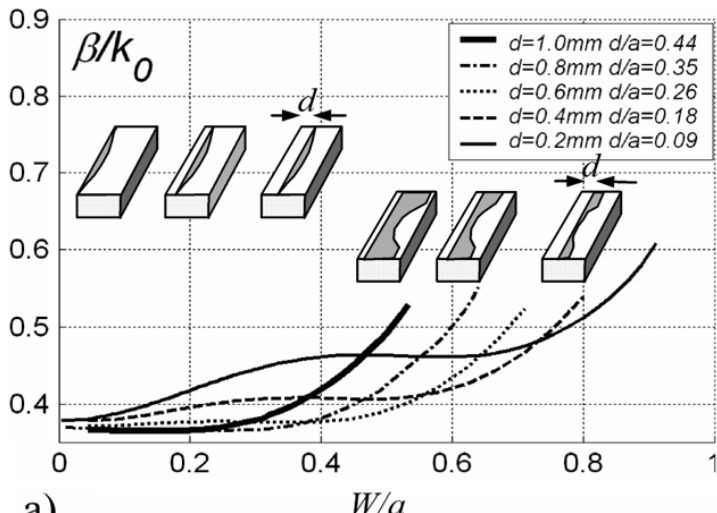

a)

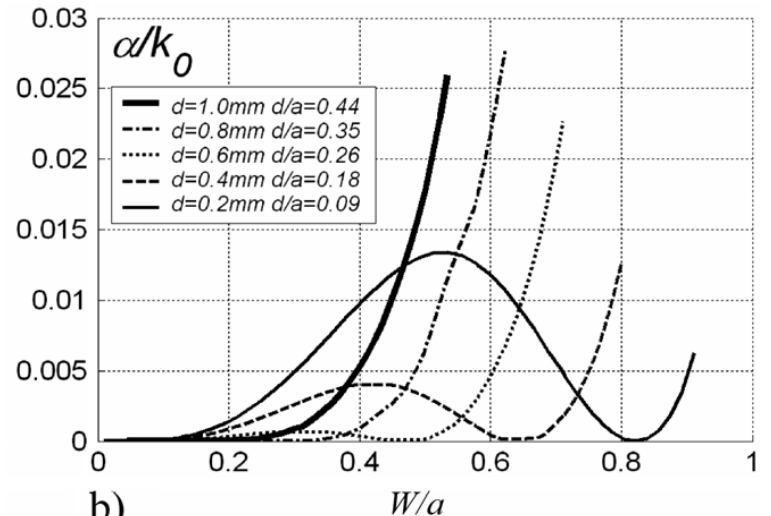

b)

Fig. 8. Width-modulated taper for the strip-type LWA (STIP-2 and STRIP-3) for different strip offsets $\left(a=2.25 \mathrm{~mm}, D=1.59 \mathrm{~mm}, \varepsilon_{r}=2.56, L=1 \mathrm{~mm}\right.$, $f=50 \mathrm{GHz}$.

vary more $\left(\Delta \beta / k_{0}=0.1\right)$ than for a narrower strip. This variation of $\beta$ is also higher than for the slot case. Moreover, the maximum reachable value of $\alpha$ has been reduced $\left(\alpha / k_{0}=0.03\right)$ to the half with respect to the slot case. Therefore, it can be said that the position-modulated taper (TAPER-1 in Fig. 1) is more suited for slot-type printed circuits than for strips.

The modulation of the width for the slot-type LWA (SLOT-2 in Fig. 1) is analyzed in Fig. 7. As commented, the slot width cannot be varied around $W=0$ (TAPER-2) since $\alpha$ cannot be made completely null. On the other hand, the strong change of $\beta$ for wide slots, which can be seen in Fig. 7(a) around $W_{0}$ (SLOT-2 and SLOT-3 in Fig. 1) also avoid a good tapering procedure. Therefore, it can be said that the slot width modulation is not a good choice to design taper LWA in hybrid quasiplanar technology.

On the contrary, the strip perturbation allows to implement both STRIP-2 and STRIP-3 taper varieties, modulating the strip width above $W=0$ and below $W=W_{0}$, as it is seen in Fig. 1 and in the inset of Fig. 8. The TAPER-2 (Fig. 3) procedure applied to the strip allows to obtain zero leakage constant when the strip width tends to zero, as it is seen in Fig. 8(b). From Fig. 8(a), it is seen that there is a tradeoff for the selection of the strip position $d$. As $d$ is decreased, the slot width can be varied in a higher range of values, from $W=0$ to $W_{\mathrm{MAX}}-(6)$ Lower values of $d$ imply a higher value of $W_{0}$ [see (6)], which makes the maximum value of $\alpha$ to increase, as seen in Fig. 8(b). 
TABLE I

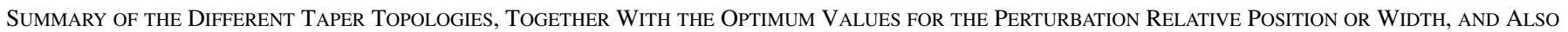
the MaXimum Reachable Leakage Constant and Variation of the Phase Constant

\begin{tabular}{c|c|c|c}
\hline Taper Type & Best range of dor W & $M A X \frac{\alpha}{k_{0}}$ & $\Delta \frac{\beta}{k_{0}}$ \\
\hline SLOT-1 & $W / a=0.4$ & 0.06 & 0.03 \\
\hline STRIP-2 & $d / a=0.18$ & 0.004 & 0.03 \\
\hline STRIP-3 & $d / a=0.18$ & 0.004 & 0.03 \\
\hline STRIP-1 & $W / a=0.62$ & 0.03 & 1 \\
\hline STRIP-4 & $d / a=0.35$ & 0.028 & 2 \\
\hline
\end{tabular}

Therefore, it is desirable to select a small value of $d$ to vary $\alpha$ in a wider range of values. For instance, $d / a=0.09$ leads to a maximum value of $\alpha / k_{0}=0.014$ in Fig. 8(b) which is achieved for $W / a=0.5$, while a more centered strip achieves a lower maximum value of leakage rate (for instance, $d / a=0.26$ gives a maximum of only $\left.\alpha / k_{0}=0.001\right)$. However, a problem arises when the strip is too close to the side waveguide wall (very small values of $d$ ). As the value of $d$ is decreased, the curve of $\beta$ suffers a stronger variation than for more centered strips (see Fig. 8(a) $\Delta \beta / k_{0}=0.1$ for $d / a=0.09$ ). This is caused by the influence of the side metal wall of the waveguide in the $\mathrm{TE}_{10}$ mode, being this perturbation stronger for strips that are closer to the side wall $(d \rightarrow 0)$. For this reason, it is better to choose a higher value of $d$, which maintains $\beta$ more constant. There is an optimum strip position which respects these two aspects. The curves for $d / a=0.18$ provide a maximum $\alpha / k_{0}=0.004$ for $W / a=0.4$ with an absolute variation in $\beta$ of $\Delta \beta / k_{0}=0.03$. With respect to the strip width modulation around $W_{0}(S T R I P-3$ and STRIP-4 in Fig. 1), the TAPER-3 procedure $\left(W<W_{0}\right)$ is better suited than the TAPER-4 $\left(W>W_{0}\right)$ due to the smaller variation of $\beta$. Although the STRIP-4 taper $\left(W>W_{0}\right)$ allows to obtain higher values for $\alpha$ than in the other taper topologies of the strip, the variation of $\beta$ is much higher $\left(\Delta \beta / k_{0}=2\right)$. For a small variation of $\beta$ in the STRIP-3 taper $\left(W<W_{0}\right), d$ cannot be chosen too small, although smaller values of $d$ provide higher ranges of variation of $\alpha$, as previously commented. Choosing $d / a=0.18$, it is obtained a small variation in $\beta\left(\Delta \beta / k_{0}=\right.$ 0.03 ) from $W_{0} / a=0.64$ to $W / a=0.4$, obtaining a maximum value of $\alpha / k_{0}=0.004$ for $W / a=0.4$.

Table I summarizes all these results, by sorting the different tapering processes in order of increasing variation of $\beta$. It must be reminded that the aim of this study is to obtain an easy tapering procedure in which only the width or the position of the printed circuit can be modulated to vary $\alpha$ while keeping $\beta$ as constant as possible in all the variation range of $\alpha$. When comparing the different printed-circuit taper topologies, it is found that the SLOT-1 taper (slot position modulation around $d=d_{0}$ ) provides the lowest perturbation in $\beta\left(\Delta \beta / k_{0}=0.03\right)$, together with the largest range of variation in $\alpha\left(\max \alpha / k_{0}=0.06\right)$, thus being selected as the best taper process. The STRIP-2 and STRIP-3 taper topologies (strip width modulation around $W=$ 0 and $W<W_{0}$, respectively), are proposed as the following best tapering mechanisms, also providing a good flat response of the phase constant $\left(\Delta \beta / k_{0}=0.03\right)$, although a smaller range of beamwidths can be obtained with them (maximum $\alpha / k_{0}=$ 0.004). Besides, Table I shows the optimum values of the per- turbation relative width or position for each taper design in order to minimize the variation of $\beta$, while maximizing the variation in $\alpha$. The fourth and fifth position in this ranking belongs to the STRIP-1 (strip position modulation) and STRIP-4 (strip width modulation with $W>W_{0}$ ), respectively. They achieve good maximums in the value of $\alpha / k_{0}$, but the variation in the phase constant is much bigger than in the previous three cases. The rest of the printed-circuit taper topologies illustrated in Fig. 1 are neglected, due either to the too strong variation in the phase constant along the antenna (SLOT-3, SLOT-4, and G-STRIP-2), or due to the inability to achieve a smooth radiation variation (SLOT-2 and G-STRIP-3 do not have a null in $\alpha / k_{0}$ ). All these results have been obtained for the desired leaky-wave mode with a constant stub height $L=1 \mathrm{~mm}$ (see sketch in Fig. 1). Next section makes a deep study in the effect of the stub height in the performance of the leaky-wave antenna.

\section{EfFect of the Parallel-Plate Stub Height in the ANTENNA PERFORMANCE}

Leaky-wave antennas based on radiation by asymmetry are loaded with a parallel-plate stub in order to reduce the influence of higher-order parallel-plate modes, which introduce cross-polarization and grating lobes in the total radiated fields [4], [8], [11]. A higher stub height improves these characteristics, but can also create mode coupling with undesired leaky-modes, which can propagate in the open waveguide structure spoiling the antenna performance [15], [16]. Therefore, the influence of the stub height must be examined for the different tapering topologies proposed in this paper. This section is devoted to the study of the effect of the stub height in both the appearance and coupling with undesired leaky-modes, and its relation with polarization purity.

The working mechanism of leaky-wave antennas is based on the excitation of a given leaky-wave mode, whose propagation and radiation properties can be controlled in order to conform to the design specifications (frequency of operation, beamwidth, beam direction, low level of secondary lobes, high polarization purity, low losses, and easy feeding mechanism). However, other modes can propagate in the open-waveguide, whose radiation properties cannot be controlled, thus being called unwanted modes. There are two important but different aspects relating to the existence of these unwanted modes in the frequency of operation of the antenna. The first is the possible excitation of any of these modes, which would spoil the performance of the antenna. For instance, if a surface-wave is excited, some part of the energy would be guided in the dielectric slab to the matched 
load, thus decreasing the antenna efficiency. If the excited undesired mode is leaky, its radiation would interfere with the radiation of the desired mode. This excitation can be avoided by using specific feeding mechanisms, which couples the energy from the source only to the wanted mode [18], or additionally by adding some modifications to the waveguide, which suppress the unwanted mode [19]. The second aspect is related to the coupling which might exist between the desired leaky-wave mode and other unwanted modes, especially with channel-guide modes [15], [20]. This phenomenon has been studied for different types of stub-loaded leaky-wave antennas, but never for the printed-circuit versions presented in this paper. Of special interest is the influence of the perturbation width or position in this unwanted coupling phenomenon, as studied in [20] for the strip-loaded groove guide LWA. This study can determine which tapering process is more likely to suffer from coupling with channel-modes, therefore spoiling the working mechanism of the tapering.

The height $L$ of the top parallel-plate waveguide (PPW) is needed to obtain a pure horizontal polarization. Higher-order PPW modes introduce radiating lobes and cross-polarization [11]. These modes are below cut-off in the air region, and their amplitude must have decayed to negligible values before reaching the radiating open end. For this reason, it is desirable to use a high stub to avoid cross polarization and grating lobes [4], [11]. However, a too long stub might allow the propagation of higher-order channel-guide modes, and a possible coupling with the desired leaky-mode. This coupling only occurs when both the phase and attenuation constants of the leaky-modes are the same [15], [20]. Fig. 9 shows the phase and attenuation constant of the desired leaky-wave mode in the different types of LWA proposed in this work (slot, strip, and grounded-strip), as a function of the normalized stub height $\left(L / \lambda_{0}\right.$, being $\lambda_{0}$ the free space wavelength) and for different printed-circuit widths.

In Fig. 9(a) and (b), it is shown the response of the slot-type LWA. For wide slots $(W / a=0.62)$, the phase and attenuation constants of the desired leaky-mode oscillates with the length $L$, as it corresponds to a standing-wave behavior at the open-end. However, for narrow slots $(W / a=0.26)$, the leaky-wave complex propagation constant moves to a nonperiodic pattern at about a length $L / \lambda_{0}=0.9$. This change in the behavior is due to the coupling with a channel-guide leaky-mode. As it is known [15], [20], channel-guide modes suffer from high leakage rates due to the fact that their energy primarily propagates in the parallel-plate region. Since the coupling between two leaky-modes can only happen when both $\beta$ and $\alpha$ of the two modes get close, those printed-circuit configuration with higher leakage rates are more likely to suffer from coupling between the desired leaky-mode and a channel-guide leaky-mode. For wide slots $(W / a=0.62$ with $d=2 \mathrm{~mm}-d / a=0.09-)$, the desired leaky-wave mode has lower leakage values $\left[\alpha / k_{0}=0.02\right.$, as depicted in Fig. 9(b)], and it is less probable to occur the coupling with a channel-guide mode. However, for the narrow slot case $(W / a=0.26$ with $d=2 \mathrm{~mm}-d / a=0.09-), \alpha / k_{0}$ has peaks above 0.1 for $L / \lambda_{0}=0.2$ [see Fig. 9(b)]. This makes the mode couple to a channel-guide mode for $L / \lambda_{0}>0.9$,

The same discussion can be applied to the rest of printed-circuit configurations. Fig. 9(c) and (d) show the behavior in the strip-type LWA, while Fig. 9(e) and (f) illustrate the response in the grounded-strip case. Again, it can be said that the higher the leakage rate $\left(\alpha / k_{0}\right)$ of the desired leaky-mode, the higher the risk to couple with a channel guide mode. Now, with narrow strips $(W / a=0.26)$, low leakage is obtained $\left(\alpha / k_{0}=0.004\right.$ for the strip and $\alpha / k_{0}=0.025$ for the grounded-strip) and no coupling occur for any antenna. However, for wide strips $(W / a=0.62)$ the grounded-strip LWA suffers a strong coupling for even shorter PPW stubs than for the slot-type LWA (we see in Fig. 9(e) and (f) that periodicity is lost for $L / \lambda_{0}>$ 0.2 ). This is due to the high value of $\alpha$ for the grounded-strip leaky-wave mode $\left[\alpha / k_{0}=0.14\right.$, see Fig. 9(f)], which makes it couple with the channel guide mode. On the contrary, the strip-type leaky-mode does not couple to any channel-mode, even for the wider strip case, due to the lower leakage rate obtained $\left[\alpha / k_{0}=0.01\right.$ in Fig. 9(d)].

As commented, a study must be made to know the minimum height of the stub $L$ needed to obtain a pure horizontal polarization while avoiding coupling with channel-guide modes. For this purpose, the full-wave method used in this paper is able to measure the interference created by higher-order PPW modes at the radiating aperture, leading to the so called normalized radiation purity [21]. The polarization efficiency can be measured as the power radiated by the main PPW mode (zero order, pure horizontal polarization with no grating lobes) normalized by the power radiated by the whole spectra of PPW modes (including higher-order PPW modes). Since all higher order PPW modes are below cut-off in the transverse direction of the vacuum medium, their amplitude decay as the observation point is moved from the dielectric interface to the PPW aperture. As the PPW height is increased, the contribution of these higher-order PPW modes is lower, obtaining a purer radiation. In [22], a deep study is made to analyze the influence of each type of printed-circuit in the radiation purity of the LWA. There, it is shown that the strip-type LWA needs a longer stub than the slot-type LWA, in order to avoid the cross polarization effect. This is due to the higher excitation of the first higher-order PPW mode in this strip-type LWA. As commented, the absence of a broad metal wall in the NRD guide makes the electric field couple to the side metal walls, thus strongly exciting the first higher-order PPW mode. In the slot-type LWA this effect is weaker, since the field lines couple to the broad metal wall of the slitted rectangular guide. It can be concluded that the strip-type LWA is less sensitive to coupling with channel-guide modes, due to its lower leakage rate. However, longer stubs than for the slot-type LWA are needed to obtain polarization purity. All these aspects must be taken into account for a practical tapered design.

\section{TAPER ANTENNA DESIGN}

All the results obtained in the last sections are needed to understand the radiation mechanism of the proposed hybrid waveguide printed-circuit leaky-wave antennas. The parametric study was obtained by solving the two-dimensional eigenvalue, in which the complex wavenumber of the different leaky-wave modes was obtained varying the geometrical parameters and frequency [10], [11]. In this section, a complete tapering procedure is presented, furnishing a longitudinal shaping of the 

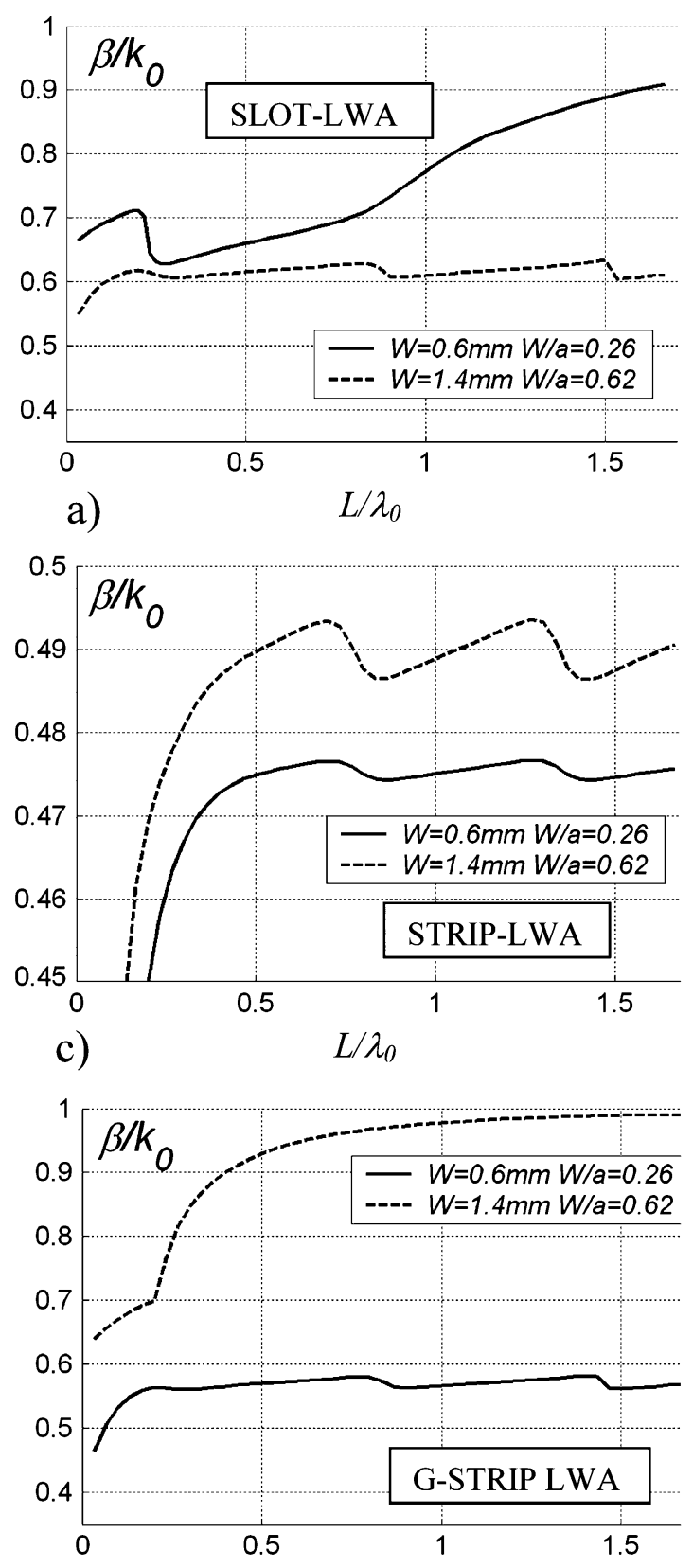

e)

$L / \lambda_{0}$
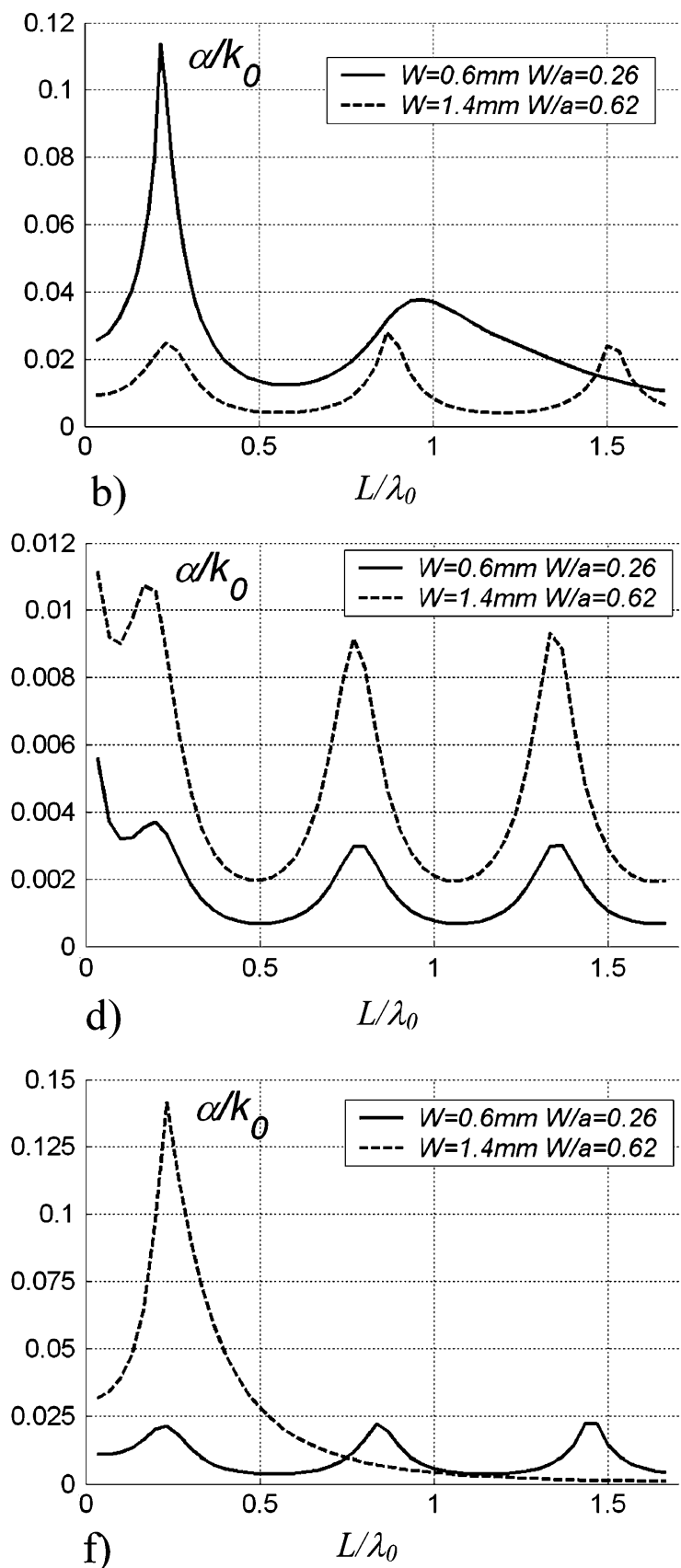

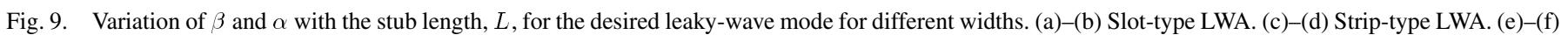
Grounded strip-type LWA ( $\left.a=2.25 \mathrm{~mm}, D=1.59 \mathrm{~mm}, \varepsilon_{r}=2.56, \boldsymbol{d}=\mathbf{0 . 2} \mathrm{mm}, f=50 \mathrm{GHz}, \lambda_{0}=6 \mathrm{~mm}\right)$.

metallization along the antenna length to obtain a cosine illumination. The design procedure for tapering LWA is well-known, and it can be found in [4]. The SLOT-1 tapering topology, selected as the best tapering mechanism in Table I, is going to be used to obtain the taper design. The variation of the leakage constant of the desired leaky-wave mode along the antenna length can be computed from the desired fields illumination $M(z)$ as follows [4]:

$\alpha\left(z=z_{0}\right)=\frac{1}{2} \frac{\left|M\left(z=z_{0}\right)\right|^{2}}{\frac{1}{\eta} \cdot \int_{z=0}^{z=L_{A}}|M(z)|^{2} \cdot \partial z-\int_{z=0}^{z=z_{0}}|M(z)|^{2} \cdot \partial z}$

where $z$ is the longitudinal coordinate of the antenna, which goes from zero to the antenna length $L_{A}$, and $\eta$ is the antenna efficiency which is determined by the amount of power radiated along the antenna length with respect to the total injected power. Introducing in (7) the desired illumination function $M(z)$ to obtain a given low-sidelobes radiation pattern, one can obtain the variation of the leakage rate. However, the phase constant of the leaky-mode, $\beta$, must be maintained unchanged in order to have all sections of the aperture radiating at the same angle $\theta_{m}$. In Section II, it was illustrated how the position of the slot can be varied for a constant slot width $(W / a=0.44)$ in order to change $\alpha$ in a wide range of values $\left(\max \alpha / k_{0}=0.06\right)$ while keeping almost unchanged $\beta\left(\Delta \beta / k_{0}=0.03\right)$. However, it is known that small phase variations may sensitively disturb the radiation pattern, being necessary an adjustment to reduce the illumination phase aberration [4], [5], [7]. This adjustment must 

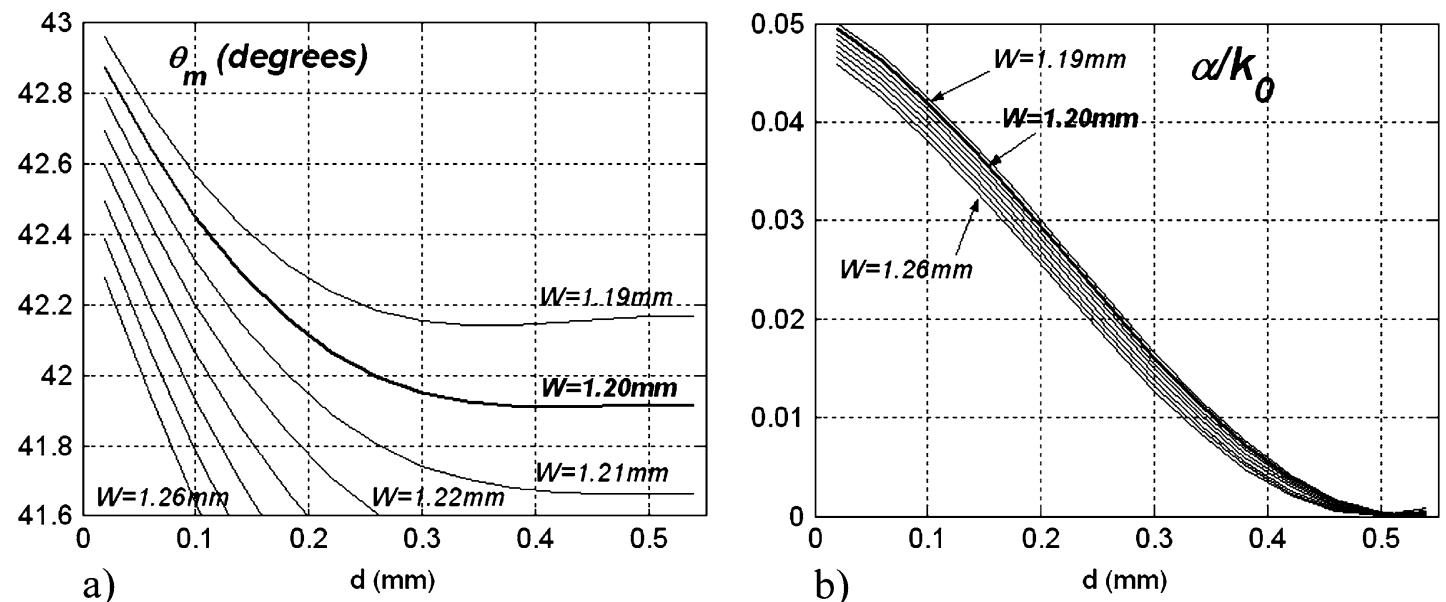

Fig. 10. Variation of phase and leakage constants of desired leaky-mode for slot-1 taper topology, varying both slot width and position $(a=2.25 \mathrm{~mm}, D=1.59$ $\mathrm{mm}, \varepsilon_{r}=2.56, \boldsymbol{L}=1 \mathrm{~mm} f=50 \mathrm{GHz}$ ).
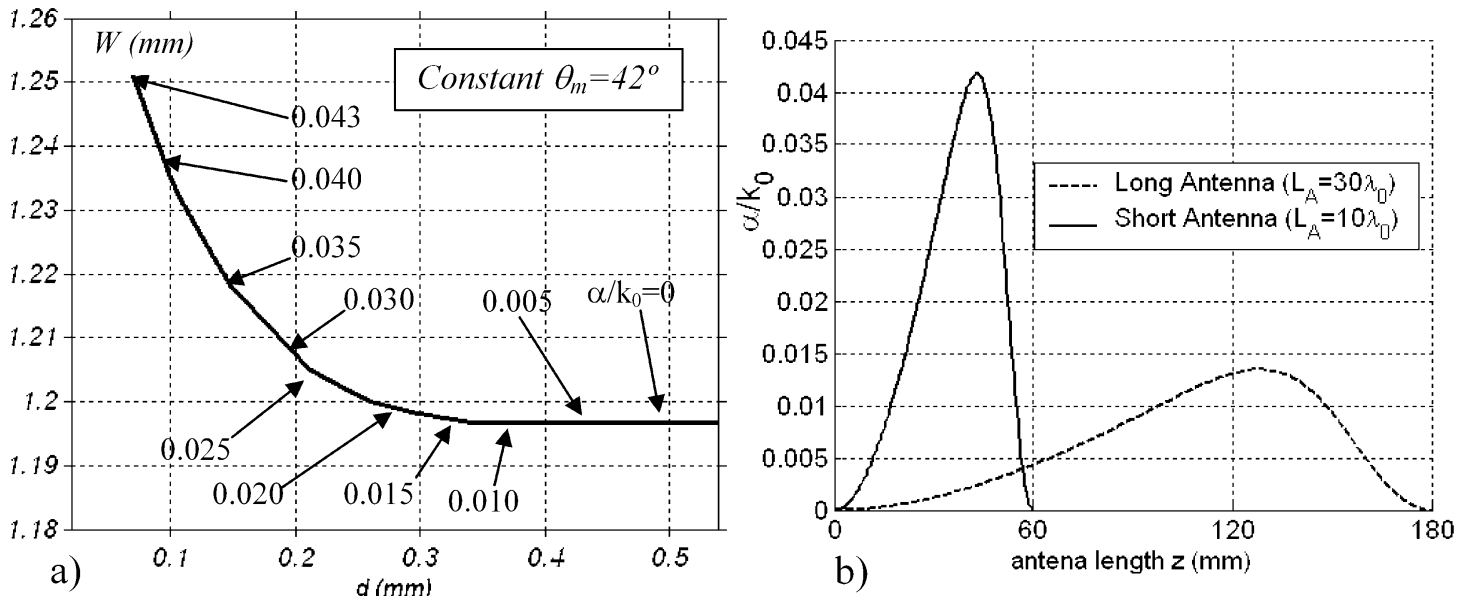

Fig. 11. (a) Slot width and position modulation to obtain a constant phase illumination for different leakage rates (b) Leakage rate variation along the antenna length for two different practical cases, long and short leaky-wave antennas ( $a=2.25 \mathrm{~mm}, D=1.59 \mathrm{~mm}, \varepsilon_{r}=2.56, L=1 \mathrm{~mm}, f=50 \mathrm{GHz}$ ).

be done by varying not only the slot position, but also the slot width. Fig. 10 illustrates this procedure, by showing the phase and the leakage constants of the desired leaky-wave mode of the slot-type LWA, by sweeping the slot position $d$ for different slot widths, around the optimum width obtained in Section II $(W=0.44 \cdot a \approx 1) \mathrm{mm}$.

The minimum change in the leakage angle of the leaky-mode $\left[\theta_{m}\right.$, Fig. 10 (a) $]$ is found for a slot width $W=1.2 \mathrm{~mm}$, which is in agreement with the summary of Table I $(W / a=0.53)$, and allows to change the leakage constant from zero to $\alpha / k_{0}=0.05$ [Fig. 10(b)], varying the slot position from $d_{0}=0.5 \mathrm{~mm}$ (centered slot) to $d=0 \mathrm{~mm}$. From $d=0.5 \mathrm{~mm}$ to $d=0.31 \mathrm{~mm}$, the phase constant does not vary at all, providing a radiating angle $\theta_{m} \approx 42^{\circ}$. However, as the slot position is further reduced from $d=0.31 \mathrm{~mm}$, the phase of the illumination changes. Fig. 11(a) shows how the slot width can be readjusted to keep $\theta_{m}$ constant, by increasing the slot width to correct the phase aberration.

This procedure is applied to obtain the curve shown in Fig. 11(a), in which a line is plotted representing the slot positions $(d)$ and widths $(W)$ for a constant beam pointing of $\theta_{m}=42^{\circ}$, and indicating the values of $\alpha / k_{0}$ from 0 to 0.043 along this line. As commented, it is seen that a constant width slot can be used up to $\alpha / k_{0}=0.015(d=0.31 \mathrm{~mm})$, keeping constant the pointing direction. For higher leakage rates, both the position and width of the slot must be modulated along the antenna length. To complete the design, it must be determined the maximum leakage rate needed. This depends basically in the desired beamwidth, which is directly related to the antenna length $L_{A}$ [see (3)]. As a result, longer antennas and lower leakage rates are needed for narrower radiated beams. Fig. 11(b) shows the leakage rate variation along the antenna length, $\alpha(z)$ [given by (7)], to obtain a taper cosine illumination function with $\eta=90 \%$ efficiency, for two different practical antennas. One antenna will be referred as the long antenna case $\left(L_{A}=30 \lambda_{0}\right)$, while the second case is that of a short antenna $\left(L_{A}=10 \lambda_{0}\right)$. From Fig. 12(b) it is seen that the short antenna needs a maximum normalized leakage rate of $\alpha / k_{0}=0.042$, while the maximum is of only $\alpha / k_{0}=0.014$ for the long one. Therefore, for the short antenna case, the slot width must be readjusted using the curve shown in Fig. 11(a), in order to correct the errors in the phase illumination. For the sections of the antenna in which $\alpha / k_{0}$ must be grater than 0.015 , the slot width is readjusted above $1.2 \mathrm{~mm}$, as illustrated in Fig. 11(a). Different normalized radiation patterns are illustrated in Fig. 12, together with the layout of the printed-circuit position-modulated slot. 

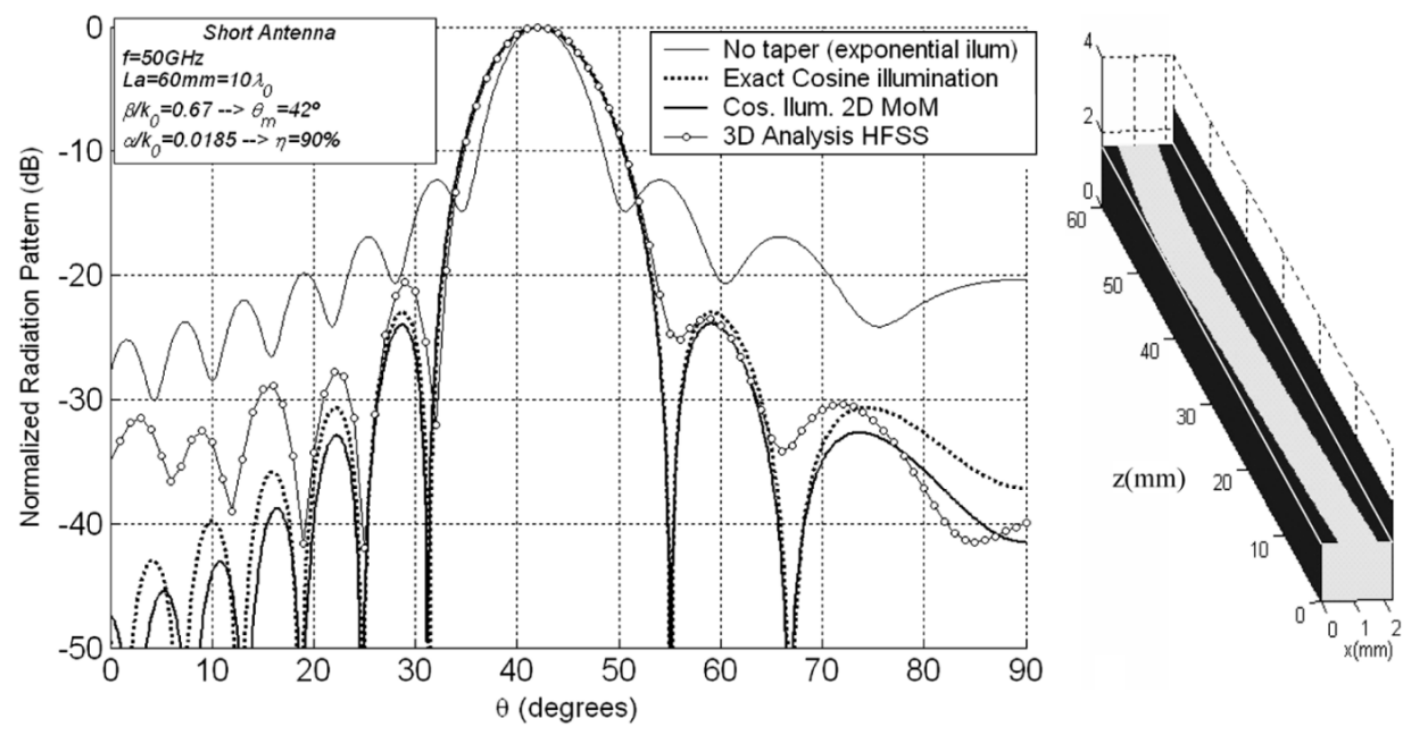

Fig. 12. Normalized Radiation Pattern obtained for the short antenna case, using the SLOT-1 taper topology and readjusting the slot width using the data shown in Fig. 15(a) $(f=50 \mathrm{GHz})$.
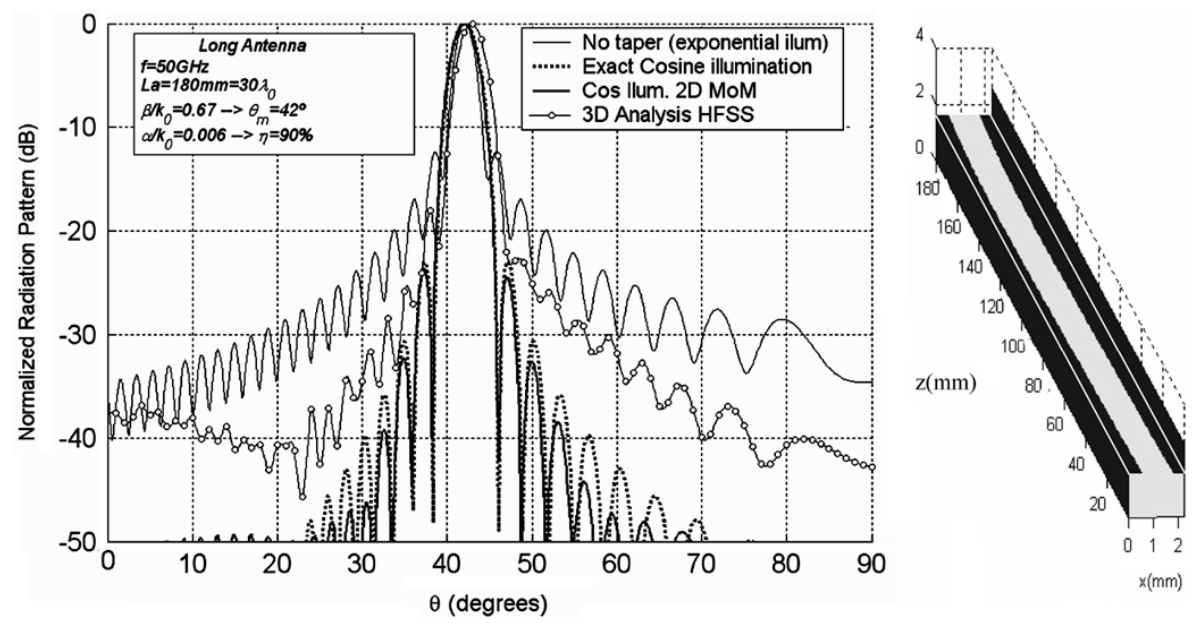

Fig. 13. Normalized Radiation Pattern obtained for the long antenna case $(f=50 \mathrm{GHz})$.

The radiation pattern obtained with a nontaper leaky-wave antenna with the same length $\left(L_{A}=10 \lambda_{0}\right.$ and exponential illumination with $\alpha / k_{0}=0.0 .185$, which provides $90 \%$ efficiency) is first plotted, to show the high sidelobes levels $(13 \mathrm{~dB})$. Also, the radiation pattern of theoretical exact cosine illumination is plotted with dotted lines to show the expected improvement in the radiation pattern ( $23 \mathrm{~dB}$ sidelobes level), at the cost of a higher beamwidth. Using the leaky-wave complex propagation constant obtained from the dimensions of the slot along the different sections of the open waveguide, $\beta(z)$ and $\alpha(z)$, the illumination predicted by the two-dimensional MoM technique can be obtained. From this illumination, the radiation pattern obtained from the taper antenna can be predicted (Fig. 12, continuous line). These results are also compared with the radiation pattern obtained from a complete three-dimensional analysis with HFSS (circles in Fig. 12). Good agreement is obtained in both cases, showing the ability of the proposed tapering technique to improve the antenna performance. Only a small difference in the pointing angle of $1^{\circ}$ is observed. The authors have checked with HFSS that this difference is due to the location of the absorbing boundary condition used by HFSS to truncate the free-space region.

Fig. 13 shows the same results but for the long antenna case. It is seen the narrower beam radiating at the same pointing angle $\left(\theta_{m}=42^{\circ}\right)$. Again, the radiation pattern predicted with the two-dimensional technique agrees very well with HFSS results. The SLOT- 1 tapering procedure allows to improve the radiation pattern. However, it was necessary to readjust the slot width to avoid the phase error. The impact of the phase error is illustrated in Fig. 14 for the two antenna cases. A theoretical linear phase error of $1^{\circ}$ and $5^{\circ}$ have been applied to the cosine illumination in order to see the effect in the radiation pattern. The effect is more visible in the long antenna than in the short one, due to the higher sensitivity of the narrower radiated beam.

Therefore, it could be thought that the correction of the phase error is more important for long antenna taper than for the short antenna case. However, due to the fact that the long antenna needs lower values of $\alpha / k_{0}$, it happens that no phase error occurs when only the position $d$ of the slot is modulated, with a constant width $W=1.2 \mathrm{~mm}$ [the maximum value of $\alpha / k_{0}=$ 

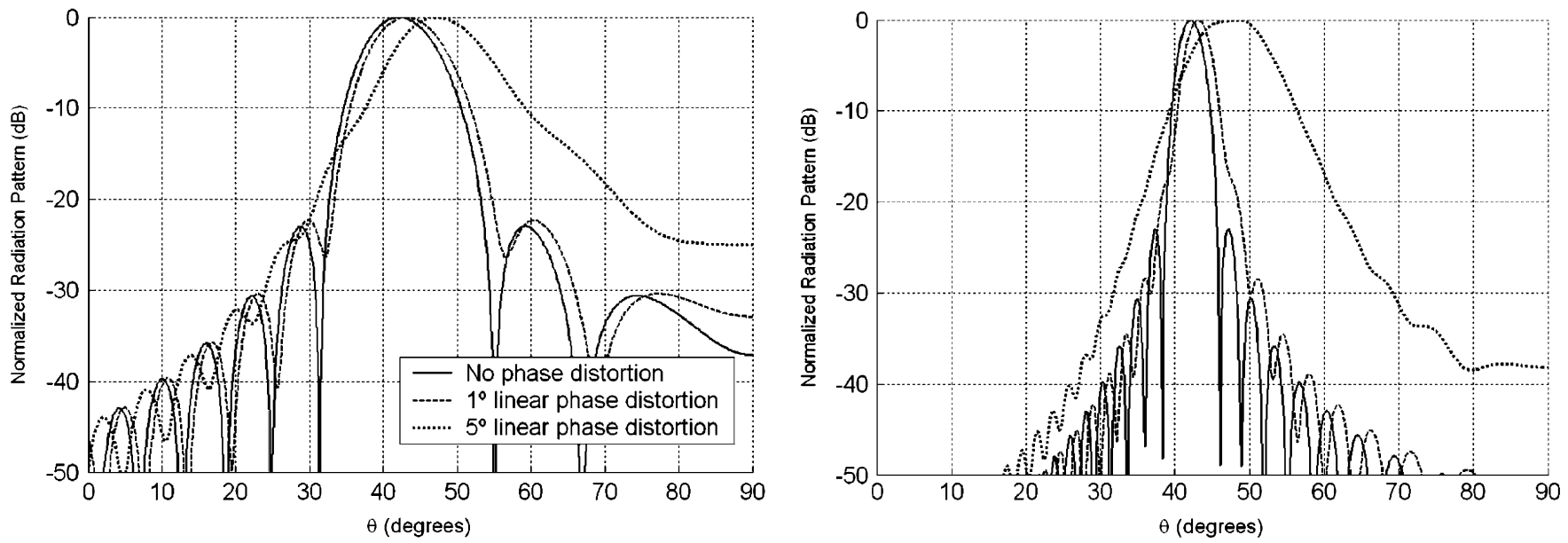

Fig. 14. Effect of the phase aberration in the radiation patterns for the long and short antenna cases.

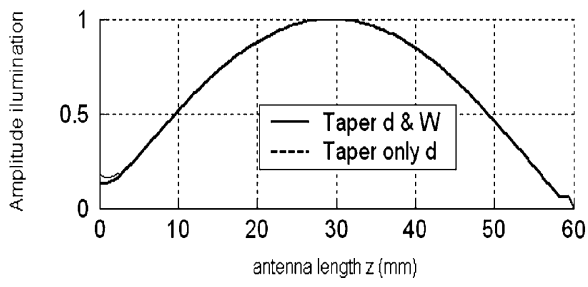

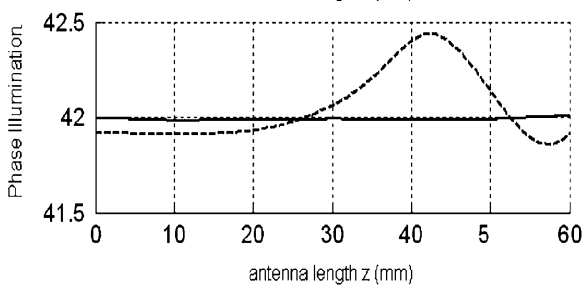

a)

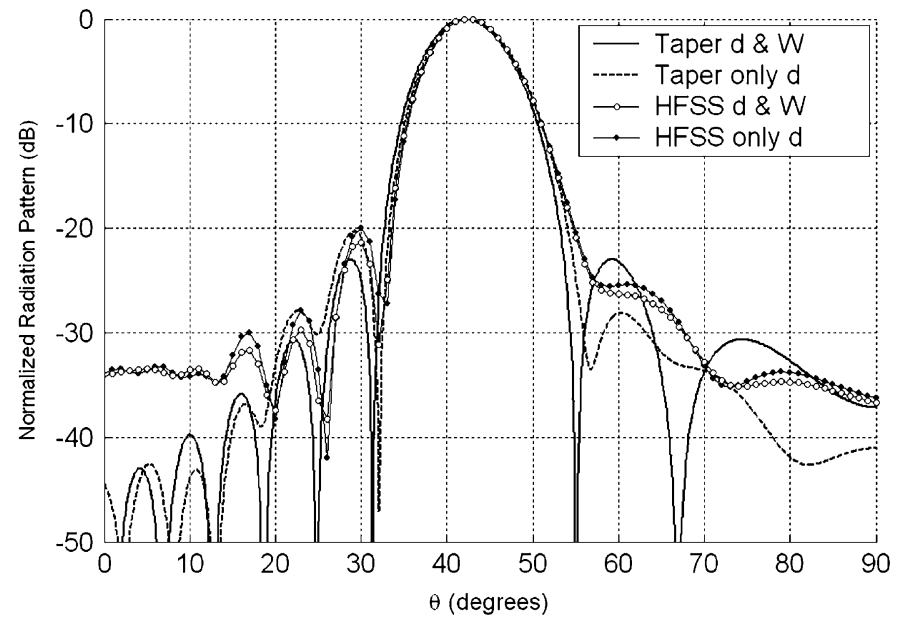

b)

Fig. 15. Differences between tapering only the slot position or both position and width for the short antenna.

0.014 lies in the horizontal part of the curves in Figs. 10(a) and 11(a)]. On the contrary, if only the position of the slot is tapered in the short antenna (with $W=1.2 \mathrm{~mm}$ ), the illumination suffers a phase aberration which can spoil the antenna performance. In this case the maximum value of $\alpha / k_{0}=0.042$ is beyond the horizontal part of Figs. 10(a) and 11(a). This effect is illustrated in Fig. 15(a), where both the amplitude and phase illumination on the parallel-plate stub aperture are shown when both $d$ and $W$ are tapered to obtain a perfect amplitude and phase illumination (continuous line) and when only the slot position $d$ is varied along the antenna (cosine amplitude illumination with some phase error shown in Fig. 15(a) with dotted line).

As it can be seen in Fig. 15(b), this phase error increases one side lobe of the radiated pattern, going from $-23 \mathrm{~dB}$ from the total taper topology (continuous line, taper $d \& W$ ) to $-20 \mathrm{~dB}$ in the case that only $d$ is tapered (dashed line). However, the degradation of the radiation pattern is not very strong due to the fact that the phase illumination error is very small $\left[0.5^{\circ}\right.$, see Fig. 15(a)] and because the short antenna is less sensitive to phase errors than the long antenna (see Fig. 14). Comparisons with HFSS analysis are also plotted to validate these results (circles). Therefore, it can be concluded that the proposed technology allows to taper the antenna by only modulating the slot position, while maintaining constant the slot width, both for short and long leaky-wave antennas. This is a good advantage with respect to other taper technologies (as [7]) for two different reasons. First, the taper design is quite easy since it only involves the variation of the slot position with a constant slot width. Second, the taper is performed in the printed circuit mask, avoiding any modification of the robust waveguide structure, where more complicate manufacturing processes are needed.

\section{CONCLUSION}

Different printed-circuit versions of asymmetrically perturbed waveguide leaky-wave antennas have been studied. These hybrid printed-waveguide antennas exhibit higher flexibility in their manufacturing process when compared to full-waveguide technology, which is of paramount importance in the millimeter-wave band. Special attention has been paid to the ability to control the leakage constant of the desired leaky-wave mode, while maintaining unchanged its phase constant. In this way, several taper configurations have been 
proposed, based on the modulation of the width or the position of the planar perturbation around certain values. It has been shown that it is important to choose the dimensions of the slot or strip perturbation, since there are optimum values to maximize the variation range of the leakage rate, while minimizing the variation in the phase constant. The slot-type LWA has shown to be better candidate than the strip-type to obtain a good offset taper topology. However, the strip type circuit allows better width tapering, both around zero width and by asymmetry control. The whole spectrum of modes of each antenna has also been studied, showing the appearance of unwanted modes, whose radiation cannot be controlled, and are therefore not suitable for antenna applications. Much care must be taken with possible coupling effects with these undesired leaky-wave modes, especially when high leakage rates are needed. The strip-type taper is less sensitive to this undesired coupling phenomenon. However, the polarization purity of the strip-type antenna is lower than for the slot case, therefore needing longer stubs to obtain pure horizontal polarization. All these aspects (tapering, coupling, polarization purity) have been studied with detail and accuracy (without any geometrical restriction) for the first time for this new type of hybrid printed-circuit waveguide leaky-wave antennas. Finally, a complete tapering procedure to obtain a cosine illumination has been applied for two different practical cases: short and long leaky-wave antennas. It has been illustrated the impact of the illumination phase aberration, showing the ability to readjust the printed circuit dimensions in order to improve the radiation pattern.

\section{ACKNOWLEDGMENT}

The authors want to thank EPSON-Ibérica foundation for its stimulating support.

\section{REFERENCES}

[1] C. H. Walter, Traveling Wave Antennas. New York: McGraw-Hill, 1965.

[2] F. J. Zucker, "Surface- and Leaky-Wave Antennas," in Antenna Engineering Handbook, 1st ed, H. Jasik, Ed. New York: McGraw-Hill, 1969 , ch. 16

[3] F. Schwering and A. A. Oliner, "Millimeter-Wave Antennas," in Antenna Handbook, Y. T. Lo and S. W. Lee, Eds. New York: Van Nostrand Reinhold, 1988, ch. 17.

[4] A. A. Oliner, "Leaky-Wave Antennas," in Antenna Engineering Handbook, 3rd ed, R. C. Johnson, Ed. New York: McGraw-Hill, 1993, ch. 10.

[5] J. A. Encinar, M. Guglielmi, and A. A. Oliner, "Taper optimization for sidelobe control in millimeter-wave metal-strip-loaded dielectric antennas," in URSI Radio Sci. Meet. Dig., Syracuse, NY, Jun. 1988, p. 397.

[6] S. Majumder and D. R. Jackson, "Radiation characteristics of one-dimensional periodic leaky-wave antennas," in AP-S Dig., vol. 1, 1997, pp. $452-455$.

[7] C. Di Nallo, F. Frezza, A. Galli, G. Gerosa, and P. Lampariello, "Stepped leaky-wave antennas for microwave and millimeter-wave applications," Ann. Télécommun., vol. 52, pp. 202-208, Mar. 1997.

[8] P. Lampariello and A. A. Oliner, "A novel phase array of printed-circuit leaky wave lines sources," in Proc. 17th European Microwave Conf., Rome, Italy, 1987, pp. 555-560.

[9] A. A. Oliner, "Scannable millimeter wave arrays," Weber Res. Inst., Polythecnic University, vol. II, Tech. Rep. poly-WRI-1543-88, Sep. 30, 1988. "A Novel Array of Printed-Circuit Uniform Leaky-Wave Line Sources".
[10] J. L. Gómez and A. A. Melcón, "Non-orthogonality relations between complex-hybrid-modes: An application for the leaky-wave analysis of laterally-shielded top-open planar transmission lines," IEEE Trans. Microwave Theory Tech., vol. 52, no. 3, pp. 760-767, Mar. 2004.

[11] — - "Radiation analysis in the space domain of laterally-shielded planar transmission lines. Part I: Theory," Radio Sci., vol. 39, pp. 1-11, Jun. 2004.

[12] G.-J. Chou and C.-K. C. Tzuang, "An integrated quasiplanar leaky-wave antenna," IEEE Trans. Microwave Theory Tech., vol. 44, no. 8, pp. 1078-1085, Aug. 1996.

[13] Z. Ma and E. Yamashita, "Space wave leakage from higher order modes on various planar transmission lines structures," in Proc. IEEE MTT-S Int. Microwave Symp., San Diego, CA, 1994, pp. 1033-1036.

[14] P. Baccarelli, P. Burghignoli, C. Di Nallo, F. Frezza, A. Galli, P. Lampariello, and G. Ruggieri, "Full-wave analysis of printed leaky-wave phase arrays," Int. J. RF Microw. Computed Aided Eng., vol. 12, pp. 272-287, May 2002.

[15] H. Shigesawa, M. Tsuji, P. Lampariello, F. Frezza, and A. A. Oliner, "Coupling between different leaky-mode types in stub-loaded leaky waveguides," IEEE Trans. Microwave Theory Tech., vol. 42, pp. 1548-1560, Aug. 1994.

[16] P. J. B. Clarricoats, P. E. Green, and A. A. Oliner, "Slot-mode propagation in rectangular waveguide," Electron, Lett., no. 2, pp. 307-308, 1966.

[17] P. Lampariello, F. Frezza, and A. A. Oliner, "The transition region between bound-wave and leaky-wave ranges for a partially dielectric-loaded open guiding structure," IEEE Trans. Microwave Theory Tech., vol. 38, no. 12, pp. 1831-1836, Dec. 1990.

[18] Y.-D. Lin, J.-W. Sheen, and C.-K. C. Tzuang, "Analysis and design of feeding structures for microstrip leaky wave antenna," IEEE Trans. Microwave Theory Tech., vol. 44, no. 9, pp. 1540-1547, Sep. 1996.

[19] R. Garg and K. C. Gupta, "Suppression of slot mode in slotted waveguide antennas," IEEE Trans. Antennas Propag., vol. 23, no. 9, pp. 730-732, 51975.

[20] Z. Ma and E. Yamashita, "Leakage characteristics of groove guide having a conductor strip," IEEE Trans. Microwave Theory Tech., vol. 42, no. 10, pp. 1925-1931, Oct. 1994.

[21] J. L. Gómez and A. A. Melcón, "Radiation analysis in the space domain of laterally-shielded planar transmission lines. Part II: Applications," Radio Sci., vol. 39, pp. 1-10, Jun. 2004.

[22] — , "Effect of the finite height of the parallel-plate stub in hybrid waveguide printed-circuit leaky-wave antennas," Tech. Univ. Cartagena, UPCT-TIC-TSC-04-01. Internal Rep.

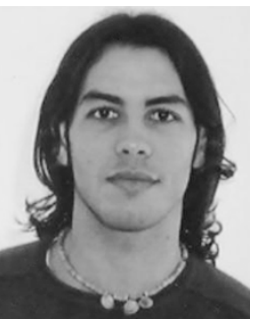

José Luis Gómez Tornero was born in Murcia, Spain, in 1977. He received the telecommunications engineer degree from the Polytechnic University of Valencia (UPV), Valencia, Spain, in 2001, and the $\mathrm{Ph} . \mathrm{D}$. degree (laurea cum laude) in telecommunication engineering from the Technical University of Cartagena (UPCT), Cartagena, Spain, in 2005.

In 1999, he joined the Radiocommunications Department, UPV, as a Research Student, where he was involved in the development of analytical and numerical tools for the study and automated design of microwave filters in waveguide technology for space applications. In 2000, he joined the Radio Frequency Division, Industry Alcatel Espacio, Madrid, Spain, where he was involved with the development of microwave active circuits for telemetry, tracking and control (TTC) transponders implicated in many different spatial missions for the European Space Agency (ESA), National Aeronautics Space Administration (NASA) and other Space Agencies. In 2001, he joined the Technical University of Cartagena, Spain, as an Assistant Professor, where he is currently developing his teaching activities. His scientific research is focused on the analysis and design of leaky-wave antennas for millimeter wave-band applications and the development of numerical methods for the analysis of novel passive radiating structures in planar and waveguide technologies. His scientific interests also include the study of active devices for microwave and millimeter wavebands, such as oscillators and active antennas.

Prof. Gómez Tornero received the second national award from the EPSONIbérica foundation for the best Ph.D. project in the field of technology of information and communications (TIC), in July 2004. 


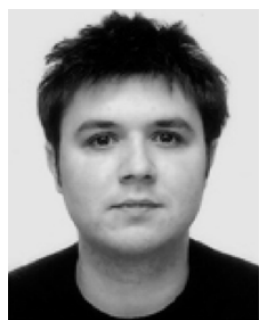

Alejandro de la Torre Martínez was born in Murcia, Spain, in 1980. He received the telecommunications engineer degree from the Polytechnic University of Cartagena (UPCT), Cartagena, Spain.

Since January 2005, he has been working in the Mobile Network Engineering Division at Siemens Company, Madrid, Spain. He and is currently awaiting a position with Siemens. His scientific interests are centred on the study of microwave and millimeter waveband systems and circuits.

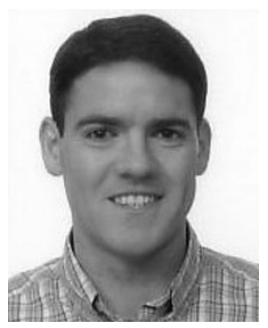

David Cañete Rebenaque (S'04) was born in Valencia, Spain, in 1976. He received the telecommunications engineer degree from the Technical University of Valencia, Valencia, Spain, in 2001, and is currently working toward the Ph.D. degree at the Technical University of Cartagena, Cartagena, Spain.

During 2001, he worked as an RF Engineer for a mobile communication company. In 2002, he joined the Communications and Information Technologies Department, Technical University of Cartagena. His research interests include the analysis and design of microwave circuits and active antennas.

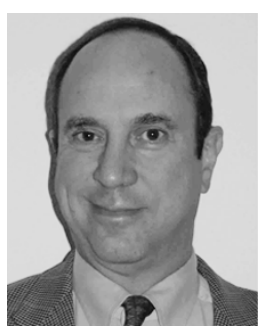

Marco Guglielmi was born in Rome, Italy, on December 17, 1954. He received the "laurea in ingegneria elettronica" degree from the University of Rome "La Sapienza," Rome, Italy, in 1979, the M.S. Degree in electrical engineering from the University of Bridgeport, Bridgeport, CT, in 1982, and the Ph.D. degree in electrophysics from the Polytechnic University, Brooklyn, NY, in 1986.

From 1984 to 1986, he was an Academic Associate and, from 1986 to 1988, he was an Assistant Professor at Polytechnic University. From 1988 to 1989, he was Assistant Professor at the New Jersey Institute of Technology, Newark, NJ. In 1989, he joined the European Space Agency as a Senior Microwave Engineer in the RF System Division of the European Space Research and Technology Centre (ESTEC), Noordwijk, The Netherlands, where he was in charge of the development of microwave filters and EM simulation tools. In 2001, he was appointed Head of the Technology Strategy Section in the Technology Programmes Department of ESTEC where he is currently contributing to the development of management processes and tools for the formulation of a European strategy for Space technology Research and Development.

Dr. Guglielmi was awarded a Fulbright Scholarship in Rome, Italy, and a Halsey International Scholarship Programme (HISP) from the University of Bridgeport, Bridgeport, CT, in 1981.

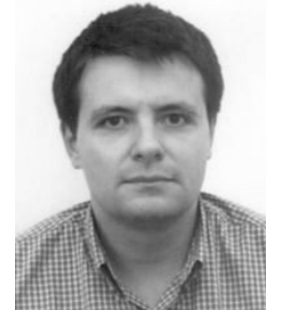

Alejandro Álvarez Melcón was born in Madrid, Spain, in 1965. He received the telecommunications engineer degree from the Polytechnic University of Madrid (UPM), Madrid, Spain, in 1991, and the Ph.D. degree in electrical engineering from the Swiss Federal Institute of Technology, Lausanne, Switzerland, in 1998

In 1988, he joined the Signal, Systems and Radiocommunications Department, UPM, as a Research Student, where he was involved in the design, testing, and measurement of broad-band spiral antennas for electromagnetic measurements support (EMS) equipment From 1991 to 1993, he was with the Radio Frequency Systems Division, European Space Agency (ESA/ESTEC), Noordwijk, The Netherlands, where he was involved in the development of analytical and numerical tools for the study of waveguide discontinuities, planar transmission lines, and microwave filters. From 1993 to 1995, he was with the Space Division, Industry Alcatel Espacio, Madrid, Spain, and he worked at the ESA, where he collaborated in several ESA/ESTEC contracts. From 1995 to 1999, he was with the Swiss Federal Institute of Technology, École Polytechnique Fédérale de Lausanne, Lausanne, Switzerland, where he worked in the field of microstrip antennas and printed circuits for space applications. In 2000, he joined the Technical University of Cartagena, Spain, where he is currently developing his teaching and research activities.

Dr. Álvarez Melcón received the Journée Internationales de Nice sur les Antennes (JINA) Best Paper Award for the best contribution to the JINA'98 International Symposium on Antennas, and the COIT/AEIT (Colegio Oficial de Ingenieros de Telecomunicación) award to the best Ph.D. thesis in basic information and communication technologies. 